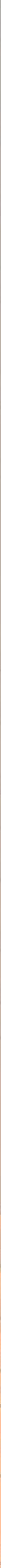




\title{
Towards local-global compatibility for Hilbert modular forms of low weight
}

\author{
James Newton
}

\begin{abstract}
We prove some new cases of local-global compatibility for the Galois representations associated to Hilbert modular forms of low weight. If $F / \mathbb{Q}$ is a totally real extension of degree $d$, we are interested in Hilbert modular forms for $F$ of weight $\left(k_{1}, \ldots, k_{d}, w\right)$, with the $k_{i}$ and $w$ odd integers and some but not all $k_{i}$ equal to 1 (the partial weight-one case). Recall that a Hecke eigenform $f$ with such a weight has an associated compatible system $\rho_{f, p}$ of two-dimensional $p$-adic representations of $\operatorname{Gal}(\bar{F} / F)$, first constructed by Jarvis using congruences to forms of cohomological weight $\left(k_{i} \geq 2\right.$ for all $\left.i\right)$.

One expects that the restriction of the representation $\rho_{f, p}$ to a decomposition group $D_{v}$ at a finite place $v \nmid p$ of $F$ should correspond (under the local Langlands correspondence) to the local factor at $v, \pi_{f, v}$, of the automorphic representation $\pi_{f}$ generated by $f$. This expectation is what we refer to as localglobal compatibility. For forms of cohomological weight, the compatibility was in most cases verified by Carayol using geometric methods. Combining this result with Jarvis's construction of Galois representations establishes many cases of local-global compatibility in the partial weight-one situation. However, when $\pi_{f, v}$ is a twist of the Steinberg representation, this method establishes a statement weaker that local-global compatibility. The difficulty in this case is to show that the Weil-Deligne representation associated to $\left.\rho_{f, p}\right|_{D_{v}}$ has a nonzero monodromy operator. In this paper, we verify local-global compatibility in many of these 'missing' cases, using methods from the $p$-adic Langlands programme (including analytic continuation of overconvergent Hilbert modular forms, maps between eigenvarieties encoding Jacquet-Langlands functoriality and Emerton's completed cohomology).
\end{abstract}

\section{Introduction}

In this paper, we study the problem of local-global compatibility for the Galois representations attached to Hilbert modular forms of low weight. These Galois representations were constructed by Jarvis [1997]. We begin by recalling the main

MSC2010: primary 11F41; secondary $11 \mathrm{~F} 33,11 \mathrm{~F} 80$.

Keywords: Hilbert modular forms, Galois representations, local-global compatibility. 
theorem of that paper. Let $F$ be a totally real finite extension of $\mathbb{Q}$. For a prime $p$, let $\iota$ be an isomorphism from a fixed algebraic closure $\overline{\mathbb{Q}}_{p}$ of $\mathbb{Q}_{p}$ to $\mathbb{C}$. If $v$ is a finite place of $F$ and $\pi_{v}$ is an admissible smooth irreducible representation of $\mathrm{GL}_{2}\left(F_{v}\right)$ over $\mathbb{C}$, we denote the Frobenius-semisimple Weil-Deligne representation associated to $\pi_{v}$ under the (Hecke normalised) local Langlands correspondence by $\sigma\left(\pi_{v}\right)$. We denote by $\sigma^{l}\left(\pi_{v}\right)$ the Weil-Deligne representation with coefficients in $\overline{\mathbb{Q}}_{p}$ obtained by composition with $\iota^{-1}$. For a continuous $\overline{\mathbb{Q}}_{p}$-representation $\rho$ of $\operatorname{Gal}\left(\bar{F}_{v} / F_{v}\right)$, with $v \nmid p$ a finite place of $F$, we denote by $\operatorname{WD}(\rho)$ the Weil-Deligne representation associated to $\rho$ by Grothendieck's $p$-adic monodromy theorem, with Frobenius semisimplification $\operatorname{WD}(\rho)^{F \text {-ss }}$ and semisimplification $\operatorname{WD}(\rho)^{\text {ss }}$ (i.e., we set the monodromy operator to zero to obtain $\left.\operatorname{WD}(\rho)^{\mathrm{ss}}\right)$.

Results of Carayol [1986b], Blasius and Rogawski [1993], Rogawski and Tunnell [1983], Taylor [1989] and Jarvis [1997] have shown the following:

Theorem 1.1. Let $\pi$ be a cuspidal algebraic ${ }^{1}$ automorphic representation of $\mathrm{GL}_{2}\left(\mathbb{A}_{F}\right)$, such that for each infinite place $\tau$ of $F$ the local factor $\pi_{\tau}$ is either discrete series or holomorphic limit of discrete series. Then there exists an irreducible representation

$$
r_{p, l}(\pi): \operatorname{Gal}(\bar{F} / F) \rightarrow \mathrm{GL}_{2}\left(\overline{\mathbb{Q}}_{p}\right)
$$

such that if $v$ is a finite place of $F$, with $v \nmid p$, and one of the following holds:

- $\pi_{\tau}$ is discrete series for all infinite places $\tau$,

- $\pi_{v}$ is not special (i.e., $\pi_{v}$ is not a twist of the Steinberg representation), then

$$
\mathrm{WD}\left(\left.r_{p, \iota}(\pi)\right|_{\mathrm{Gal}\left(\bar{F}_{v} / F_{v}\right)}\right)^{F-\mathrm{ss}} \cong \sigma^{\iota}\left(\pi_{v}\right) .
$$

Remark 1.2. The irreducibility of the Galois representation appearing in this theorem is proved using an argument of Ribet [1977] (see [Taylor 1995, Proposition 3.1]).

Remark 1.3. In the excluded case, where $\pi_{\tau}$ is holomorphic limit of discrete series for some $\tau$ and $\pi_{v}$ is special, then Jarvis [1997, Remark 7.3] proved that

$$
\mathrm{WD}\left(\left.r_{p, l}(\pi)\right|_{\mathrm{Gal}\left(\bar{F}_{v} / F_{v}\right)}\right)^{\mathrm{sS}} \cong \sigma^{\iota}\left(\pi_{v}\right)^{\mathrm{ss}} .
$$

The main result of this paper addresses some of the excluded cases in Theorem 1.1. We prove:

Theorem 1.4. Let $\pi$ be as in Theorem 1.1, suppose that $\pi_{\tau}$ is holomorphic limit of discrete series for some infinite place $\tau$ and let $v \nmid p$ be a finite place of $F$ such that $\pi_{v}$ is special.

Suppose the following technical hypotheses hold:

\footnotetext{
${ }^{1}$ By algebraic, we just mean that the usual parity condition on the weights holds.
} 
(1) The prime $p$ is absolutely unramified in $F$.

(2) For every place $w$ of $F$ with $w \mid p, \pi_{w}$ is an unramified principal series representation.

(3) Moreover, for each $w \mid p$ we have $\pi_{w} \cong \operatorname{Ind}\left(\mu_{1} \otimes \mu_{2}\right)$ (normalised parabolic induction from a Borel subgroup) with $\mu_{1}, \mu_{2}$ distinct unramified characters of $F_{w}^{\times}$.

(4) The residual representation

$$
\bar{r}_{p, l}(\pi): \operatorname{Gal}(\bar{F} / F) \rightarrow \mathrm{GL}_{2}\left(\overline{\mathbb{F}}_{p}\right)
$$

is irreducible.

Then

$$
\mathrm{WD}\left(\left.r_{p, l}(\pi)\right|_{\mathrm{Gal}\left(\bar{F}_{v} / F_{v}\right)}\right)^{F-\mathrm{ss}} \cong \sigma^{\iota}\left(\pi_{v}\right) .
$$

Remark 1.5. We will not discuss the question of local-global compatibility at places dividing $p$ in this paper. However, under the ' $p$-distinguished' hypothesis (3) made above, an argument using analytic continuation of crystalline periods (as done in [Jorza 2010] for the case of low-weight Siegel modular forms) shows that the representations $r_{p, l}(\pi)$ are crystalline at places dividing $p$, with the expected associated Weil-Deligne representation.

The main novelty in this theorem is that we are able to establish nontriviality of the monodromy operator for a Galois representation which is only indirectly related (by congruences) to the cohomology of Shimura varieties.

1.6. The technical hypotheses. We will say a few words about the hypotheses in Theorem 1.4. Hypotheses (1) and (2) are satisfied for all but finitely many $p$. Hypothesis (1) appears because we use results of Kassaei [2013] on analytic continuation and gluing of overconvergent Hilbert modular forms. At least for forms of parallel weight, results have been announced by Pilloni and Stroh [2013] and Sasaki [2014] which apply without restriction on the ramification of $p$, so it seems reasonable to hope that this hypothesis could be removed in future. Once hypothesis (1) is removed, hypothesis (2) can be substantially relaxed by first making a base change to an extension $F^{\prime} / F$ in which $p$ ramifies.

Hypothesis (3) is again present in [Kassaei 2013] and has been removed by Pilloni and Stroh [2013] and Sasaki [2014] in their setting, but we also make some independent use of this condition. In particular, we use (3) to keep track of the distinct $p$-stabilisations when we apply $p$-adic functoriality to move between different kinds of $p$-adic automorphic form. By contrast, in [Pilloni and Stroh 2013; Sasaki 2014], the situation is that the various $p$-stabilisations form a single generalised eigenspace for the Hecke operators. So extending our techniques to this setting would require more care when applying $p$-adic functoriality. 
We assume hypothesis (4) in order to apply the results of [Newton 2013] (it simplifies the analysis of completed cohomology of Shimura curves). It should be possible to avoid this assumption by modifying the arguments of that paper to work with overconvergent cohomology [Ash and Stevens 2008].

To summarise, we believe that the techniques described in this paper could in principle prove a version of the above theorem with just one technical hypothesis: for each place $w \mid p$ of $F, \pi_{w}$ is not a twist of the Steinberg representation. To handle the remaining cases seems to require a new idea or a different method.

Remark 1.7. Recent work of Kassaei, Sasaki and Tian [Kassaei et al. 2014] permits us to relax hypotheses (2) and (3) slightly (allowing tame ramification). We have kept to the 'unramified' setting for expository reasons, but here is the precise condition which can replace hypotheses (2) and (3) in Theorem 1.4:

(2') For every place $w$ of $F$ with $w \mid p, \pi_{w} \cong \operatorname{Ind}\left(\mu_{1} \otimes \mu_{2}\right)$ with $\mu_{1}, \mu_{2}$ distinct characters of $F_{w}^{\times}$whose ratio is tamely ramified.

1.8. Sketch of proof. We now sketch the proof of Theorem 1.4. By twisting and base change we may assume that $[F: \mathbb{Q}]$ is even, that $\pi_{v}$ is an unramified twist of the Steinberg representation, and that we have an auxiliary finite place $w$ such that $\pi_{w}$ is also an unramified twist of Steinberg. In light of Remark 1.3 we just need to show that the Weil-Deligne representation associated to the local representation

$$
\rho_{v}=\left.r_{p, \iota}(\pi)\right|_{\operatorname{Gal}\left(\bar{F}_{v} / F_{v}\right)}
$$

has a nonzero monodromy operator. Equivalently, we must show that $\rho_{v}$ is not an unramified representation. We will assume that $\rho_{v}$ is unramified and obtain a contradiction.

The auxiliary Steinberg place $w$ allows us to find systems of Hecke eigenvalues attached to $\pi$ in the $p$-adically completed cohomology of Shimura curves associated to indefinite quaternion algebras. In this context, a $p$-adic analogue of Mazur's principle [Newton 2013, Theorem 4.33] allows us to show that, since the representation $\rho_{v}$ is unramified, we can strip $v$ from the level of $\pi$. More precisely we can produce overconvergent Hilbert modular forms, with level prime to $v$, which share the same system of Hecke eigenvalues (outside $v$ ) as $\pi$. Finally, a generalisation, due to Kassaei, of Buzzard and Taylor's gluing and analytic continuation of overconvergent eigenforms allows us to produce a classical Hilbert modular form, with level prime to $v$, contributing to $\pi$. But we assumed that $\pi_{v}$ was (a twist of) Steinberg, so $\pi$ contains no nonzero $\mathrm{GL}_{2}\left(\mathrm{O}_{F_{v}}\right)$-invariant vectors. Therefore we obtain the desired contradiction.

Readers familiar with the theory of $p$-adic and overconvergent automorphic forms may find it amusing that we make use of three different avatars of overconvergent automorphic forms in this paper - firstly geometrically defined overconvergent Hilbert 
modular forms (sections of automorphic line bundles on strict neighbourhoods of the ordinary locus in Hilbert modular varieties as in, for example, [Kisin and Lai 2005]), secondly the overconvergent automorphic forms on definite quaternion algebras defined by Buzzard [2007] and finally the spaces obtained by applying Emerton's locally analytic Jacquet functor to the $p$-adically completed cohomology of Shimura curves [Emerton 2006b; Newton 2013].

The organisation of our paper is as follows: In Section 2 we reduce the statement of Theorem 1.4 to a special case. Then, in Section 3, we describe the various eigenvarieties we will make use of, together with their construction. We next explain how to find systems of Hecke eigenvalues arising from the automorphic representations $\pi$ of interest in the completed cohomology of Shimura curves, where we apply the level optimisation results of [Newton 2013] (Section 4). Finally we return to overconvergent modular forms on Hilbert modular varieties and apply a crucial result of Kassaei [2013] (Section 5).

1.9. Other approaches. Luu $[2015, \S 3.2]$ has also recently described an approach to proving some cases of local-global compatibility for Hilbert modular forms of low weight when $\pi$ satisfies an ordinarity hypothesis at places dividing $p$. We will use the same notation as in the previous subsection. Using the assumption that $\rho_{v}$ is unramified, Luu applied a modularity lifting theorem to produce an ordinary $p$-adic Hilbert modular form $g$ with level prime to $v$ and the same system of Hecke eigenvalues (outside $v$ ) as $\pi$, and then imposed a hypothesis that amounts to ruling out the existence of $g$. It may also be possible to show that $g$ is classical (hence obtaining a contradiction without an additional hypothesis) using a variant of the methods applied in the parallel weight-one case (as in, for example, [Kassaei 2013]), but it is not obvious to the author that these methods can be easily applied. One obstacle is that, unlike the parallel weight-one case, not all the $p$-stabilisations of the newform generating $\pi$ are ordinary. The advantage of our method is that these $p$-stabilisations still have finite slope, so we can apply the level optimisation results of [Newton 2013] to obtain several overconvergent forms and then apply [Kassaei 2013].

1.10. Examples. Until recently, not many examples of Hilbert modular forms of partial weight one were known - in particular, as far as the author knows, the only known examples were CM forms (and therefore not twists of Steinberg at any place). However, Moy and Specter [2015] have recently explicitly computed examples which are non-CM and Steinberg at a finite place.

\section{Notation and reductions}

We let $F / \mathbb{Q}$ be a totally real, finite extension of $\mathbb{Q}$, and fix a prime $p$ such that $p$ is absolutely unramified in $F$. We denote by $\Sigma$ the set of Archimedean places of $F$. 
We write $\mathbb{A}_{F}$ for the adeles of $F$ and $\mathbb{A}_{F}^{\infty}$ for the finite adeles. For a finite place $v$ of $F$ we write $O_{v}$ for the ring of integers in the local field $F_{v}$. For convenience, we fix once and for all a choice of uniformiser $\varpi_{v} \in \mathcal{O}_{v}$ for each finite place $v$.

We denote by $\Sigma_{p}$ the set of embeddings $F \hookrightarrow \overline{\mathbb{Q}}_{p}$, and for a place $\mathfrak{p} \mid p$ denote by $\Sigma_{\mathfrak{p}}$ those embeddings which extend to $F_{\mathfrak{p}}$. Our fixed isomorphism $\iota: \overline{\mathbb{Q}}_{p} \cong \mathbb{C}$ induces a bijection between $\Sigma$ and $\Sigma_{p}$.

We will need some notation for (limits of) discrete series representations of $\mathrm{GL}_{2}(\mathbb{R})$. For $k \geq 2$ and $w$ integers of the same parity, we let $D_{k, w}$ denote the discrete series representation of $\mathrm{GL}_{2}(\mathbb{R})$ with central character $t \mapsto t^{-w}$ defined in [Carayol 1986b, Section 0.2]. For $k=1$ and $w$ an odd integer, we define $D_{1, w}$ to be the limit of discrete series $\operatorname{Ind}(\mu, v)$, where the induction is a normalised parabolic induction from a Borel subgroup and $\mu, v$ are characters of $\mathbb{R}^{\times}$defined by $\mu(t)=|t|^{-w / 2} \operatorname{sgn}(t)$ and $v(t)=|t|^{-w / 2}$.

We assume $\pi$ is an automorphic representation of $\mathrm{GL}_{2}\left(\mathbb{A}_{F}\right)$ as in the statement of Theorem 1.4, and that $v$ is a finite place of $F$ with $\pi_{v}$ special. First we note that by twisting and making a quadratic base change (to an extension of $F$ in which $v$ splits) we can assume without loss of generality that $[F: \mathbb{Q}]$ is even, that $\pi_{v}$ is an unramified twist of the Steinberg representation and that there is another finite place $v^{\prime}$ with $\pi_{v^{\prime}}$ also an unramified twist of Steinberg.

With this in mind, for the rest of the paper we fix a cuspidal automorphic representation $\pi_{0}$ of $\mathrm{GL}_{2}\left(\mathbb{A}_{F}\right)$ as in the statement of Theorem 1.4, and moreover assume that:

- $[F: \mathbb{Q}]$ is even.

- $\pi_{0, v}$ is an unramified twist of Steinberg.

- There is a finite place $v^{\prime}$ coprime to $p$ and $v$ such that $\pi_{0, v^{\prime}}$ is an unramified twist of Steinberg.

- $\pi_{0, \tau} \cong D_{k_{\tau}, w}$ for each $\tau \in \Sigma$ with $k_{\tau}$ an odd positive integer and $w$ an odd integer (independent of $\tau$ ).

2.1. Hilbert modular forms and varieties. We now proceed to reduce Theorem 1.4 to a statement about Hilbert modular forms. Let $L \subset \overline{\mathbb{Q}}_{p}$ be a finite extension of $\mathbb{Q}_{p}$ which contains the image of every embedding from $F$ into $\overline{\mathbb{Q}}_{p}$. Let $\mathfrak{n}$ and $\mathfrak{m}$ be two coprime ideals of $O_{F}$, both coprime to $p$, and suppose that $\mathfrak{n}$ is divisible by a rational integer which is $\geq 4$.

Definition 2.2. Let $\mathfrak{c}$ be a fractional ideal of $F$ such that its absolute norm $N \mathfrak{c}$ is coprime to $\mathfrak{m n}$. We denote by $\underline{\mathscr{M}}^{\mathfrak{c}}(\mathfrak{n}, \mathfrak{m})$ the functor from schemes over $\mathrm{O}_{L}$ to sets taking $S$ to isomorphism classes of tuples $(A, \lambda,[\eta])$, where:

- $A$ is a Hilbert-Blumenthal abelian scheme over $S$ (in particular $A$ is equipped with an action of $O_{F}$ [Kisin and Lai 2005, 1.1]). 
- $\lambda$ is a c-polarisation of $A$ [Kisin and Lai 2005, 1.2].

- $\eta$ is an $\mathbb{O}_{F}$-equivariant closed immersion of $S$-group schemes

$$
\eta:\left(\mathbb{O}_{F} / \mathfrak{m n}\right)^{\vee} \hookrightarrow A[\mathfrak{m n}]
$$

and $[\eta]$ is its equivalence class under the natural action of $\left(\mathbb{O}_{F} / \mathfrak{m}\right)^{\times}$. Here $\left(\mathrm{O}_{F} / \mathfrak{m n}\right)^{\vee}$ denotes the Cartier dual of the constant $S$-group scheme (with ${ }_{F}$ action) $\mathbb{O}_{F} / \mathfrak{m n}$.

Under our assumptions, the functor $\underline{\mathscr{M}}^{\mathfrak{c}}(\mathfrak{n}, \mathfrak{m})$ is representable by a smooth $\mathcal{O}_{L}$-scheme $\mathscr{M}^{\mathfrak{c}}(\mathfrak{n}, \mathfrak{m})$, and there is a good theory of toroidal and minimal compactifications (see [loc. cit., 1.6, 1.8] — although we use a slightly modified level structure, everything in this reference goes through).

Having fixed a choice of representatives $\mathfrak{c}_{1}, \ldots, \mathfrak{c}_{h}$ for the narrow class group of $F$ (which we may assume all satisfy $N_{\mathfrak{c}}$ coprime to $p \mathfrak{n m}$ ), we define $\mathscr{M}(\mathfrak{n}, \mathfrak{m})$ to be the disjoint union of the $\mathscr{M}^{\mathfrak{c}_{i}}(\mathfrak{n}, \mathfrak{m})$.

For $\kappa$ a homomorphism

$$
\kappa: \operatorname{Res}_{\mathbb{Z}}^{\mathbb{O}_{F}} \mathbb{G}_{m / \mathscr{O}_{L}} \rightarrow \mathbb{G}_{m / \mathscr{O}_{L}}
$$

we have line bundles $\underline{\omega}^{\kappa}$ as in [loc. cit., 1.4.2] on $\mathscr{M}(\mathfrak{n}, \mathfrak{m})$, which extend to suitable toroidal compactifications. We assume that $w$ is an integer such that the character $N^{w} \cdot \kappa^{-1}$ admits a square root. Then, following [loc. cit., 1.11], we define Hecke operators $T_{\mathfrak{a}}$ for each prime ideal $\mathfrak{a}$ of $\mathscr{O}_{F}$ coprime to $\mathfrak{m} \mathfrak{n}$ and $U_{\mathfrak{a}}$ for each prime ideal $\mathfrak{a}$ dividing $\mathfrak{m} \mathfrak{n}$.

Denote by $\mathbb{T}(\mathfrak{n}, \mathfrak{m})$ the polynomial algebra over $\mathbb{Z}$ generated by symbols $T_{\mathfrak{a}}$ and $U_{\mathfrak{a}}$ as above. This algebra naturally acts on $H^{0}\left(\mathscr{M}(\mathfrak{n}, \mathfrak{m})_{L}, \underline{\omega}^{\kappa}\right)$, and we say that a nonzero element $f$ of this $L$-vector space is a Hecke eigenform if the action of $\mathbb{T}(\mathfrak{n}, \mathfrak{m})$ preserves the one-dimensional space $L \cdot f$. If $f$ is a cuspidal Hecke eigenform, we denote by $\theta_{f}$ the character $\mathbb{T}(\mathfrak{n}, \mathfrak{m}) \rightarrow L$ giving the action of the Hecke algebra on $f$. After projecting to a classical Hilbert modular form (as described in [loc. cit., 1.11.8]) $f$ generates (a model over $L$ for) the finite part $\pi_{f}^{\infty}$ of a cuspidal automorphic representation $\pi_{f}$ of $\operatorname{GL}_{2}\left(\mathbb{A}_{F}\right)$. For $\tau \in \Sigma$ we have $\pi_{\tau}=D_{k_{\tau}, w}$, where the $k_{\tau}$ can be read off from $\kappa$ (using the bijection between $\Sigma$ and $\Sigma_{p}$ ). The central character of $\pi_{f}$ is an algebraic Hecke character of the form $\chi_{f} \omega^{-w}$, where $\omega$ is the norm character and $\chi_{f}$ is a finite-order character. The Galois representation $r_{\pi_{f}, \iota}$ is the unique semisimple representation, unramified outside $p \mathfrak{m} \mathfrak{n}$, such that for $\mathfrak{q} \nmid p \mathfrak{m} \mathfrak{n}$ a geometric Frobenius element at $\mathfrak{q}$ has characteristic polynomial $X^{2}-\theta_{f}\left(T_{\mathfrak{q}}\right) X+(N \mathfrak{q})^{1-w_{\iota}} \iota^{-1} \chi_{f}\left(\varpi_{\mathfrak{q}}\right)$

In the subsequent sections of the paper, we will prove the following 'level optimisation' result: 
Proposition 2.3. Suppose $\mathfrak{m}=\mathfrak{q l}$ is a product of two prime ideals. Let $f \in$ $H^{0}\left(\mathscr{M}(\mathfrak{n}, \mathfrak{m})_{L}, \underline{\omega}^{\kappa}\right)$ be a Hecke eigenform. Suppose that:

- The local factor $\pi_{f, \mathfrak{q}}$ is an unramified twist of the Steinberg representation.

- The residual representation

$$
\bar{r}_{p, l}\left(\pi_{f}\right): \operatorname{Gal}(\bar{F} / F) \rightarrow \mathrm{GL}_{2}\left(\overline{\mathbb{F}}_{p}\right)
$$

is irreducible.

- The polynomials $X^{2}-\theta_{f}\left(T_{\mathfrak{p}}\right) X+(N \mathfrak{p})^{1-w_{\iota}} \iota^{-1} \chi_{f}\left(\varpi_{\mathfrak{p}}\right)$ have distinct roots for all prime ideals $\mathfrak{p} \mid p$.

- The local representation

is unramified.

$$
\left.r_{p, \iota}\left(\pi_{f}\right)\right|_{\mathrm{Gal}\left(\bar{F}_{\mathrm{l}} / F_{\mathfrak{l}}\right)}
$$

Then there exists a Hecke eigenform $g \in H^{0}\left(\mathscr{M}(\mathfrak{n}, \mathfrak{q})_{L}, \underline{\omega}^{\kappa}\right)$ such that the Hecke eigenvalues of $f$ and $g$ coincide outside $\mathfrak{l}$ and $\theta_{f}\left(U_{\mathfrak{l}}\right)$ is one of the roots of $X^{2}-\theta_{g}\left(T_{\mathfrak{l}}\right) X+(N \mathfrak{l})^{1-w_{\iota}-1} \chi_{f}\left(\varpi_{\mathfrak{l}}\right)$.

We now explain why this proposition is sufficient to deduce Theorem 1.4.

Lemma 2.4. Proposition 2.3 implies Theorem 1.4.

Proof. Recall that we have reduced the statement of Theorem 1.4 to the case of $\pi_{0}$ as described immediately before Section 2.1. We denote the finite part of $\pi_{0}$ by $\pi_{0}^{\infty}$. For $\mathfrak{m}$ any nonzero ideal of $\mathcal{O}_{F}$ we define congruence subgroups $U_{0}(\mathfrak{m})$ and $U_{1}(\mathfrak{m})$ to be the subgroups of $\mathrm{GL}_{2}(\widehat{\mathbb{Z}})$ given by

$$
\begin{aligned}
& U_{0}(\mathfrak{m})=\left\{g \in \mathrm{GL}_{2}(\hat{\mathbb{Z}}): g=\left(\begin{array}{ll}
* & * \\
0 & *
\end{array}\right) \bmod \mathfrak{m}\right\} \\
& U_{1}(\mathfrak{m})=\left\{g \in \mathrm{GL}_{2}(\hat{\mathbb{Z}}): g=\left(\begin{array}{ll}
* & * \\
0 & 1
\end{array}\right) \bmod \mathfrak{m}\right\}
\end{aligned}
$$

Now we denote by $\mathfrak{l}$ and $\mathfrak{q}$ the prime ideals of $\mathfrak{O}_{F}$ corresponding to the places $v$ and $v^{\prime}$ respectively, and suppose that $\mathfrak{n}$ is an ideal of $O_{F}$, coprime to $p \mathfrak{q l}$ and

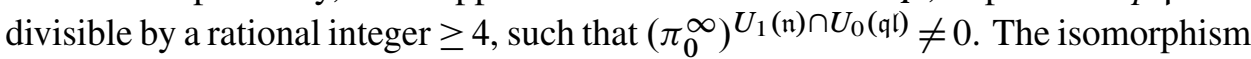
$\iota: \overline{\mathbb{Q}}_{p} \rightarrow \mathbb{C}$ induces a bijection between $\Sigma$ and the embeddings $\Sigma_{p}$ from $F \hookrightarrow L$, so using this we can associate a character

$$
\kappa: \operatorname{Res}_{\mathbb{Z}}^{\mathbb{O}_{F}} \mathbb{G}_{m / O_{L}} \rightarrow \mathbb{G}_{m / \mathbb{O}_{L}}
$$

to the $\Sigma$-tuple of integers $\left\{k_{\tau}\right\}_{\tau \in \Sigma}$ describing the Archimedean part of $\pi_{0}$. Now (possibly enlarging $L$ ) there is a Hecke eigenform $f \in H^{0}\left(\mathscr{M}(\mathfrak{n}, \mathfrak{q} \mathfrak{l})_{L}, \underline{\omega}^{\kappa}\right)$ with $\pi_{f} \cong \pi_{0}$, and so $f$ satisfies the hypotheses of Proposition 2.3. This proposition gives us a Hecke eigenform $g \in H^{0}\left(\mathscr{M}(\mathfrak{n}, \mathfrak{q})_{L}, \underline{\omega}^{\kappa}\right)$ with $\pi_{g} \cong \pi_{0}$, and so the space 
$\left(\pi_{0}^{\infty}\right)^{U_{1}(\mathfrak{n}) \cap U_{0}(\mathfrak{q})}$ is nonzero, which contradicts the assumption that $\pi_{0, \mathfrak{l}}$ is a twist of Steinberg.

We end this section by discussing Hilbert modular varieties with Iwahori level at $p$ and a definition of overconvergent Hilbert modular forms.

Definition 2.5. Let $\mathfrak{c}$ be a fractional ideal of $F$ such that its absolute norm $N \mathfrak{c}$ is coprime to $p \mathfrak{m n}$. We denote by $\mathscr{M}^{\mathrm{c}, \mathrm{Iw}}(\mathfrak{n}, \mathfrak{m})$ the functor from schemes over $O_{L}$ to sets taking $S$ to isomorphism classes of tuples $(A, \lambda,[\eta], H)$ up to isomorphism, where $(A, \lambda,[\eta])$ are as in Definition 2.2 and $H$ is a finite flat subgroup scheme of $A[p]$, stable under the action of $\mathbb{O}_{F}$, of rank $p^{[F: \mathbb{Q}]}$ and isotropic with respect to the $\lambda$-Weil pairing.

The functor $\mathscr{M}^{\mathfrak{c}, \mathrm{Iw}}(\mathfrak{n}, \mathfrak{m})$ is represented by an $\mathscr{O}_{L}$-scheme $\mathscr{M}^{\mathrm{c}, \mathrm{Iw}}(\mathfrak{n}, \mathfrak{m})$. We denote by $\mathscr{M}^{\mathrm{Iw}}(\mathfrak{n}, \mathfrak{m})$ the disjoint union of these over suitable representatives $\mathfrak{c}_{i}$ of the narrow class group, as before.

Denoting the rigid generic fibre of $\mathscr{M}^{\mathrm{Iw}}(\mathfrak{n}, \mathfrak{m})$ by $M^{\mathrm{Iw}}(\mathfrak{n}, \mathfrak{m})_{L}$, we can consider sections of the line bundles $\underline{\omega}^{\kappa}$ over strict neighbourhoods of the locus in $M^{\mathrm{Iw}}(\mathfrak{n}, \mathfrak{m})_{L}$, where $H$ is Cartier dual to $\mathcal{O}_{F} / p$ (i.e., the multiplicative ordinary locus), to obtain a space $M_{\kappa}^{\dagger}(\mathfrak{n}, \mathfrak{m})$ of overconvergent modular forms of weight $\kappa$ (see [Kassaei 2013, §4]).

\section{Eigenvarieties}

We will need to make use of eigenvarieties constructed in different contexts. To clarify the relationship between these eigenvarieties, we are going to follow the abstract approach of [Bellaïche and Chenevier 2009, 7.2]. First we need to discuss the weight spaces over which our eigenvarieties will live.

3.1. Weight spaces. We set $G=\operatorname{Res}_{F / \mathbb{Q}}\left(\mathrm{GL}_{2}\right)\left(\mathbb{Q}_{p}\right)=\prod_{\mathfrak{p} \mid p} G_{\mathfrak{p}}$, where $G_{\mathfrak{p}}=$ $\mathrm{GL}_{2}\left(F_{\mathfrak{p}}\right)$. We denote by $B=\prod_{\mathfrak{p} \mid p} B_{\mathfrak{p}} \subset G$ the Borel subgroup comprising uppertriangular matrices and by $T=\prod_{\mathfrak{p} \mid p} T_{\mathfrak{p}}$ the maximal torus comprising diagonal matrices. We denote by $N_{\mathfrak{p}}$ the subgroup of $B_{\mathfrak{p}}$ whose elements have 1 on the diagonal. Finally, $T_{0}=\prod_{\mathfrak{p} \mid p} T_{0, \mathfrak{p}} \subset T$ is the compact subgroup given by elements with integral entries.

Fix a finite extension $L \subset \overline{\mathbb{Q}}_{p}$ of $\mathbb{Q}_{p}$, which we assume contains a normal closure of $F$. The functor taking an $L$-affinoid $\operatorname{Sp} A$ to the set of continuous $A^{\times}$-valued characters of

$$
T_{0}=\left(\mathscr{O}_{F} \otimes_{\mathbb{Z}} \mathbb{Z}_{p}\right)^{\times} \times\left(\mathscr{O}_{F} \otimes_{\mathbb{Z}} \mathbb{Z}_{p}\right)^{\times}
$$

is representable by a rigid analytic space $\widehat{T}_{0}$ over $L$ [Buzzard 2004, §2]. Likewise, we have a rigid space $\hat{T}$ representing continuous characters of $T$. 
Definition 3.2. Denote by $\mathscr{W}$ the subspace of $\widehat{T}_{0}$ whose points correspond to continuous characters of $T_{0}$ which are trivial on a finite-index subgroup of $\mathrm{O}_{F}^{\times}$ (embedded diagonally in $T_{0}$ ).

Suppose we have an algebraic character $\kappa: \operatorname{Res}_{\mathbb{Z}}^{\mathbb{O}_{F}} \mathbb{G}_{m} / \mathscr{O}_{L} \rightarrow \mathbb{G}_{m} / \mathscr{O}_{L}$ such that $N^{w} \cdot \kappa^{-1}$ admits a square root. Recall that $\Sigma_{p}$ denotes the set of embeddings from $F$ to $L$, so $\kappa$ corresponds to a $\Sigma$-tuple of integers $\left\{k_{\tau}\right\}_{\tau \in \Sigma}$, with the same parity as $w$.

The character

$$
\left(t_{1}, t_{2}\right) \mapsto \prod_{\tau \in \Sigma_{p}} \tau\left(t_{1}\right)^{\frac{k_{\tau}-2-w}{2}} \tau\left(t_{2}\right)^{\frac{-w-k_{\tau}+2}{2}}
$$

is a point in $\mathscr{W}(L)$, which we also denote by $\kappa$.

Definition 3.3. Denote by $\mathscr{W}_{\kappa}$ the subspace of $\mathscr{W}$ such that maps from an $L$-affinoid $\operatorname{Sp} A$ to $\mathscr{W}_{\kappa}$ correspond to characters $\left(\chi_{1}, \chi_{2}\right):\left(\mathcal{O}_{F} \otimes_{\mathbb{Z}} \mathbb{Z}_{p}\right)^{\times} \times\left(\mathscr{O}_{F} \otimes_{\mathbb{Z}} \mathbb{Z}_{p}\right)^{\times} \rightarrow A^{\times}$ such that $\chi_{1} \chi_{2}=\mathrm{Nm}^{-w}$ and $\chi_{1} \chi_{2}^{-1}=\kappa \mathrm{Nm}^{-2} \cdot(\epsilon \circ \mathrm{Nm})$, where $\epsilon$ is a continuous character $\epsilon: \mathbb{Z}_{p}^{\times} \rightarrow A^{\times}$with

$$
v_{p}(1-\epsilon(a))>\frac{1}{p-1}
$$

for all $a \in \mathbb{Z}_{p}^{\times}$. Here Nm is the natural extension of the norm map to a continuous $\operatorname{map}\left(\mathcal{O}_{F} \otimes_{\mathbb{Z}} \mathbb{Z}_{p}\right)^{\times} \rightarrow \mathbb{Z}_{p}^{\times}$.

Taking $\epsilon$ to be the trivial character, we see that $\kappa$ is a point of $\mathscr{W}_{\kappa}$. In fact, $\mathscr{W}_{\kappa}$ is a (one-dimensional) disc in $\mathscr{W}$, with centre $\kappa$.

Remark 3.4. The weight space $\mathscr{W}$ is isomorphic to the weight space (also denoted by $\mathscr{W})$ defined in [Buzzard 2007, §8]. Our character $\left(\chi_{1}, \chi_{2}\right)$ of $\left(\mathscr{O}_{F} \otimes_{\mathbb{Z}} \mathbb{Z}_{p}\right)^{\times} \times$ $\left(0_{F} \otimes_{\mathbb{Z}} \mathbb{Z}_{p}\right)^{\times}$corresponds to the character $(n, v)=\left(\chi_{1} \chi_{2}^{-1}, \chi_{2}\right)$ in Buzzard's weight space.

The weight space $\mathscr{W}_{\kappa}$ is isomorphic to the weight space (also denoted by $\mathscr{W}_{\kappa}$ ) defined in [Kisin and Lai 2005, §4.5].

We are going to build eigenvarieties interpolating classical Hilbert modular forms over the weight space $\mathscr{W}_{\kappa}$. These eigenvarieties will be constructed using three different notions of 'overconvergent automorphic form'.

3.5. Refinements. For $\mathfrak{p} \mid p$, denote by $I_{\mathfrak{p}}$ the Iwahori subgroup of $G_{\mathfrak{p}}$, comprising matrices which reduce to upper-triangular matrices mod $\varpi_{\mathfrak{p}}$. We write $I$ for the product $\prod_{\mathfrak{p} \mid p} I_{\mathfrak{p}} \subset G$. 
Definition 3.6. Let $\pi_{\mathfrak{p}}$ be an irreducible smooth representation of $G_{\mathfrak{p}}$ on a complex vector space which is either an irreducible principal series or a twist of the Steinberg representation. An accessible refinement of $\pi_{\mathfrak{p}}$ is a character $\chi$ of $T_{\mathfrak{p}}$ such that there is a $G_{\mathfrak{p}}$-equivariant embedding

$$
\pi_{\mathfrak{p}} \hookrightarrow \operatorname{Ind}_{B_{\mathfrak{p}}}^{G_{\mathfrak{p}}} \chi .
$$

Remark 3.7. We have the following explicit description of accessible refinements: we have $\pi_{\mathfrak{p}} \cong \operatorname{Ind}_{B_{\mathfrak{p}}}^{G_{\mathfrak{p}}} \mu_{1} \otimes \mu_{2}$ or $\pi_{\mathfrak{p}} \cong \mathrm{St} \otimes \mu$, where $\mu, \mu_{i}$ are characters of $F_{\mathfrak{p}} \times$.

- Suppose $\pi_{\mathfrak{p}} \cong \operatorname{Ind}_{B_{\mathfrak{p}}}^{G_{\mathfrak{p}}} \mu_{1} \otimes \mu_{2}$. Then the accessible refinements of $\pi_{\mathfrak{p}}$ are the characters $\mu_{1} \otimes \mu_{2}$ and $\mu_{2} \otimes \mu_{1}$. Note that if $\mu_{1}=\mu_{2}$ these refinements are the same.

- Suppose $\pi_{\mathfrak{p}} \cong \mathrm{St} \otimes \mu$. Then $\pi_{\mathfrak{p}}$ is isomorphic to the unique irreducible subrepresentation of the normalised induction $\operatorname{Ind}_{B_{\mathfrak{p}}}^{G_{\mathfrak{p}}} \mu|\cdot|_{\mathfrak{p}}^{1 / 2} \otimes \mu|\cdot|_{\mathfrak{p}}^{-1 / 2}$ and the unique accessible refinement of $\pi_{\mathfrak{p}}$ is $\mu|\cdot|_{\mathfrak{p}}^{1 / 2} \otimes \mu|\cdot|_{\mathfrak{p}}^{-1 / 2}$.

For an ideal $\mathfrak{n}$ of $\mathscr{O}_{F}$, coprime to $p$, we denote by $\mathscr{H}(\mathfrak{n})$ the free commutative polynomial ring over $\mathbb{Z}$ on generators labelled $T_{v}$ and $S_{v}$ for places $v$ of $F$ not dividing $p \mathfrak{n}$ and $U_{v}$ for places $v \mid \mathfrak{n}$.

For $\mathfrak{m}, \mathfrak{n}$ ideals of $\mathfrak{O}_{F}$ (coprime to each other and to $p$ ), suppose $\pi$ is a cuspidal automorphic representation of $\mathrm{GL}_{2}\left(\mathbb{A}_{F}\right)$ with $\left(\pi^{\infty p}\right)^{U_{1}(\mathfrak{n}) \cap U_{0}(\mathfrak{m})} \neq 0$ and $\pi_{\tau}=$ $D_{k_{\tau}, w}$ for each $\tau \in \Sigma$, with $k_{\tau} \geq 2$ and $k_{\tau}=w \bmod 2$ for each $\tau$. We say that such a $\pi$ is a classical automorphic representation of tame level $U_{1}(\mathfrak{n}) \cap U_{0}(\mathfrak{m})$ and weight $(k, w)$. We moreover say that $\pi$ has finite slope if the smooth Jacquet modules $J_{B_{\mathfrak{p}}}\left(\pi_{\mathfrak{p}}\right)$ are nonzero for all $\mathfrak{p} \mid p$. Equivalently, the $\pi_{\mathfrak{p}}$ are all irreducible principal series or twists of Steinberg (since local factors of a cuspidal automorphic representation are generic).

Definition 3.8. Suppose $\pi$ is a finite-slope representation. An accessible refinement of $\pi$ is a character

$$
\chi=\bigotimes_{\mathfrak{p} \mid p} \chi_{\mathfrak{p}}: T \rightarrow \mathbb{C}^{\times}
$$

such that each $\chi_{\mathfrak{p}}$ is an accessible refinement of $\pi_{\mathfrak{p}}$.

Remark 3.9. The possible accessible refinements of finite-slope automorphic representations are completely classified by Remark 3.7.

Definition 3.10. We say that a finite-slope representation is unramified if $\pi_{\mathfrak{p}}$ is either an unramified principal series or an unramified twist of Steinberg for all $\mathfrak{p} \mid p$.

Remark 3.11. The following two conditions are easily seen to be equivalent to $\pi$ being unramified:

- For every $\mathfrak{p} \mid p, \pi_{\mathfrak{p}}$ has nonzero invariants for the Iwahori subgroup $I_{\mathfrak{p}}$. 
- Every accessible refinement of $\pi$ factors through $T / T_{0}$.

Definition 3.12. Suppose $\pi$ is a finite-slope representation of weight $(k, w)$ and $\chi$ is an accessible refinement of $\pi$. We define a continuous character

$$
v(\pi, \chi): T \rightarrow \overline{\mathbb{Q}}_{p}^{\times}
$$

by

$$
v(\pi, \chi)=\bigotimes_{\mathfrak{p} \mid p} \iota^{-1} \chi_{\mathfrak{p}}|\cdot|_{\mathfrak{p}}^{1 / 2} \otimes|\cdot|_{\mathfrak{p}}^{-1 / 2} \prod_{\tau \in \Sigma_{\mathfrak{p}}} \tau^{\left(k_{\tau}-2-w\right) / 2} \otimes \tau^{\left(-w-k_{\tau}+2\right) / 2} .
$$

Remark 3.13. Our discussion of accessible refinements and the definition of the character $v(\pi, \chi)$ is parallel to that of [Chenevier 2009, §1.4]. We will see in Section 4.2 that these definitions are completely natural when constructing eigenvarieties using completed cohomology and Emerton's locally analytic Jacquet functor.

\subsection{Abstract eigenvarieties.}

Definition 3.15. Let $\mathscr{W}^{\prime}$ be any subspace of $\mathscr{W}$ which is an admissible increasing union of affinoids (in practice it will be $\mathscr{W}_{\kappa}$ ). Let $\mathscr{H}$ be a commutative $\mathbb{Z}$-algebra and let $\mathscr{Z}$ be a subset of $\operatorname{Hom}\left(\mathscr{H}, \overline{\mathbb{Q}}_{p}\right) \times \widehat{T}\left(\overline{\mathbb{Q}}_{p}\right)$ whose image in $\widehat{T}_{0}\left(\overline{\mathbb{Q}}_{p}\right)$ is an accumulation $^{2}$ and Zariski-dense subset of $\mathscr{W}^{\prime}$. Denote by $Y$ the fibre product of $\widehat{T}$ and $\mathscr{W}^{\prime}$ over $\widehat{T}_{0}$. Then an eigenvariety for the triple $\left(\mathscr{H}, \mathscr{Z}, \mathscr{W}^{\prime}\right)$ is a reduced rigid space $X$ over $L$ equipped with:

- a ring homomorphism $\psi: \mathscr{H} \rightarrow \mathscr{O}(X)$,

- a finite morphism $v: X \rightarrow Y$,

- an accumulation and Zariski-dense subset $Z \subset X\left(\overline{\mathbb{Q}}_{p}\right)$ (which we refer to as the 'classical subset'),

such that the following are satisfied:

(1) For all open affinoids $V \subset Y$ the natural map

$$
\psi \otimes v^{*}: \mathscr{H} \otimes \mathcal{O}(V) \rightarrow \mathcal{O}\left(v^{-1}(V)\right)
$$

is surjective.

(2) The natural evaluation map

$$
\begin{aligned}
X\left(\overline{\mathbb{Q}}_{p}\right) & \rightarrow \operatorname{Hom}\left(\mathscr{H}, \overline{\mathbb{Q}}_{p}\right), \\
x & \mapsto \psi_{x}:=(h \mapsto \psi(h)(x))
\end{aligned}
$$

induces a bijection $z \mapsto\left(\psi_{z}, v(z)\right)$ from $Z$ to $\mathscr{Z}$.

${ }^{2} \mathrm{~A}$ set is accumulation if each point $z \in Z$ has a basis of affinoid neighbourhoods $U$ such that $Z \cap U$ is Zariski-dense in $U$. 
The key property of the above definition is that an eigenvariety is unique up to unique isomorphism, by [Bellaïche and Chenevier 2009, Proposition 7.2.8].

Remark 3.16. An alternative way to abstractly characterise eigenvarieties in our context is as Zariski closures of sets of classical points in the rigid space given by the product of the rigid generic fibre of a Galois (pseudo)deformation ring and some affine spaces or copies of $\mathbb{G}_{m}$ (to keep track of additional Hecke eigenvalues).

For $\mathfrak{m}, \mathfrak{n}$ ideals of $\mathscr{O}_{F}$ (coprime to each other and to $p$ ), suppose $\pi$ is an unramified representation of tame level $U_{1}(\mathfrak{n}) \cap U_{0}(\mathfrak{m})$.

There is a natural action of $\mathscr{H}(\mathfrak{m n})$ on $\left(\pi^{\infty}\right)^{U_{1}(\mathfrak{n}) \cap U_{0}(p \mathfrak{m})}$, where we let $T_{v}$ and $U_{v}$ act by double coset operators

$$
\left[U\left(\begin{array}{cc}
\varpi_{v} & 0 \\
0 & 1
\end{array}\right) U\right]
$$

and let $S_{v}$ act by the double coset operator

$$
\left[U\left(\begin{array}{cc}
\varpi_{v} & 0 \\
0 & \varpi_{v}
\end{array}\right) U\right]
$$

where $U=U_{1}(\mathfrak{n}) \cap U_{0}(p \mathfrak{m})$.

Given such a $\pi$, we obtain a subset $\mathscr{Z}(\mathfrak{n}, \mathfrak{m})_{\pi}$ of $\operatorname{Hom}\left(\mathscr{H}(\mathfrak{m n}), \overline{\mathbb{Q}}_{p}\right) \times \hat{T}\left(\overline{\mathbb{Q}}_{p}\right)$ by taking pairs

$$
(\psi, v(\pi, \chi))
$$

where $\chi$ is an accessible refinement of $\pi$ and $\psi$ is a character corresponding (via $\iota$ ) to a Hecke eigenform in $\left(\pi^{\infty}\right)^{U_{1}(\mathfrak{n}) \cap U_{0}(p \mathfrak{m})}$. Note that any element of the set $\mathscr{Z}(\mathfrak{n}, \mathfrak{m})_{\pi}$ determines $\pi$, by strong multiplicity one. The choice of accessible refinement $\chi$ corresponds to a choice of $U_{\mathfrak{p}}$-eigenvalue in the space $\left(\pi_{\mathfrak{p}}\right)^{I_{\mathfrak{p}}}$ for each $\mathfrak{p} \mid p$. The character $\kappa_{\pi}:=\left.v(\pi, \chi)\right|_{T_{0}}$ is independent of the refinement $\chi$.

Definition 3.17. Let $\mathfrak{m}$ and $\mathfrak{n}$ be a pair of coprime ideals in $\mathfrak{O}_{F}$, both coprime to $p$.

(1) Denote by $\mathscr{Z}(\mathfrak{n}, \mathfrak{m})$ the union of the $\mathscr{Z}(\mathfrak{n}, \mathfrak{m})_{\pi}$ obtained from unramified $\pi$ with tame level $U_{1}(\mathfrak{n}) \cap U_{0}(\mathfrak{m})$ such that $\kappa_{\pi} \in \mathscr{W}_{\kappa}$.

(2) For $\mathfrak{q}$ a prime divisor of $\mathfrak{m}$, we write $\mathscr{Z}(\mathfrak{n}, \mathfrak{m})^{\mathfrak{q} \text {-sp }}$ for the subset of $\mathscr{Z}(\mathfrak{n}, \mathfrak{m})$ arising from $\pi$ with $\pi^{U_{1}(\mathfrak{n}) \cap U_{0}(p \mathfrak{m} / \mathfrak{q})}=0$ (equivalently, the local factor $\pi_{\mathfrak{q}}$ is an unramified twist of the Steinberg representation).

(3) Similarly, we write $\mathscr{Z}(\mathfrak{n}, \mathfrak{m})^{\mathfrak{q} \text {-ps }}$ for the subset of $\mathscr{Z}(\mathfrak{n}, \mathfrak{m})$ arising from $\pi$ with $\pi^{U_{1}(\mathfrak{n}) \cap U_{0}(p \mathfrak{m} / \mathfrak{q})} \neq 0$ (equivalently, the local factor $\pi_{\mathfrak{q}}$ is an unramified principal series representation). 
(4) Now we consider the irreducible mod $p$ Galois representation $\bar{\rho}=\bar{r}_{p, l}\left(\pi_{f}\right)$. We can define $\mathscr{Z}(\mathfrak{n}, \mathfrak{m})_{\bar{\rho}}, \mathscr{Z}(\mathfrak{n}, \mathfrak{m})_{\bar{\rho}}^{\mathfrak{q} \text {-sp }}$ and $\mathscr{Z}(\mathfrak{n}, \mathfrak{m})_{\bar{\rho}}^{\mathfrak{q}-p s}$ to be the subsets of $\mathscr{Z}(\mathfrak{n}, \mathfrak{m})$, etc. arising from those $\pi$ with residual Galois representation $\bar{r}_{p, l}(\pi)$ isomorphic to $\bar{\rho}$.

\subsection{Buzzard's eigenvarieties.}

Theorem 3.19. There exist eigenvarieties $\mathscr{E}(\mathfrak{n}, \mathfrak{m})_{\bar{\rho}}, \mathscr{E}(\mathfrak{n}, \mathfrak{m})_{\bar{\rho}}^{\mathfrak{q}-\mathrm{sp}}$ and $\mathscr{E}(\mathfrak{n}, \mathfrak{m})_{\bar{\rho}}^{\mathfrak{q}-\mathrm{ps}}$ for the triples

$$
\begin{aligned}
& \left(\mathscr{H}(\mathfrak{m n}), \mathscr{Z}(\mathfrak{n}, \mathfrak{m})_{\bar{\rho}}, \mathscr{W}_{\kappa}\right), \\
& \left(\mathscr{H}(\mathfrak{m n}), \mathscr{Z}(\mathfrak{n}, \mathfrak{m})_{\bar{\rho}}^{\mathfrak{q}-\mathrm{sp}}, \mathscr{W}_{\kappa}\right), \\
& \left(\mathscr{H}(\mathfrak{m n}), \mathscr{Z}(\mathfrak{n}, \mathfrak{m})_{\bar{\rho}}^{\mathfrak{q}-\mathrm{ps}}, \mathscr{W}_{\kappa}\right) .
\end{aligned}
$$

We denote the classical subsets of these eigenvarieties by $Z(\mathfrak{n}, \mathfrak{m})_{\bar{\rho}}, Z(\mathfrak{n}, \mathfrak{m})_{\bar{\rho}}^{\mathfrak{q} \text {-sp }}$ and $Z(\mathfrak{n}, \mathfrak{m})_{\bar{\rho}}^{\mathfrak{q}-\mathrm{ps}}$. The following properties are satisfied by these eigenvarieties:

- There are closed immersions

$$
\mathscr{E}(\mathfrak{n}, \mathfrak{m})_{\bar{\rho}}^{\mathfrak{q}-\mathrm{sp}} \hookrightarrow \mathscr{E}(\mathfrak{n}, \mathfrak{m})_{\bar{\rho}} \quad \text { and } \quad \mathscr{E}(\mathfrak{n}, \mathfrak{m})_{\bar{\rho}}^{\mathfrak{q}-\mathrm{ps}} \hookrightarrow \mathscr{E}(\mathfrak{n}, \mathfrak{m})_{\bar{\rho}}
$$

commuting with the maps to weight space and respecting the homomorphisms $\psi$ from $\mathscr{H}(\mathfrak{m n})$, with images $X, Y$, respectively, given by unions of irreducible components (in the sense of [Conrad 1999]).

- Each irreducible component of $\mathscr{E}(\mathfrak{n}, \mathfrak{m})_{\bar{\rho}}$ is contained in precisely one of $X$ and $Y$.

- We have $Z(\mathfrak{n}, \mathfrak{m})_{\bar{\rho}} \cap X=Z(\mathfrak{n}, \mathfrak{m})_{\bar{\rho}}^{\mathfrak{q}-\mathrm{sp}}$ and $Z(\mathfrak{n}, \mathfrak{m})_{\bar{\rho}} \cap Y=Z(\mathfrak{n}, \mathfrak{m})_{\bar{\rho}}^{\mathfrak{q}-\mathrm{ps}}$.

- There is a map

$$
\mathscr{E}(\mathfrak{n}, \mathfrak{m})_{\bar{\rho}}^{\mathfrak{q}-p s} \rightarrow \mathscr{E}(\mathfrak{n}, \mathfrak{m} / \mathfrak{q})_{\bar{\rho}}
$$

surjective on closed points, for which the preimage of a closed point $x \in$ $\mathscr{E}(\mathfrak{n}, \mathfrak{m} / \mathfrak{q})_{\bar{\rho}}$ is indexed by the roots of the Hecke polynomial $X^{2}-\psi\left(T_{\mathfrak{q}}\right)(x) X+$ $N \mathfrak{q} \psi\left(S_{\mathfrak{q}}\right)(x)$.

Proof. We fix a definite quaternion algebra $D / F$, ramified precisely at the infinite places of $F$, and an isomorphism $\left(D \otimes_{F} \mathbb{A}_{F}^{\infty}\right)^{\times} \cong \mathrm{GL}_{2}\left(\mathbb{A}_{F}^{\infty}\right)$. Buzzard's definition [2007, Part III] of overconvergent automorphic forms on $D$, with tame level $U_{1}(\mathfrak{n}) \cap U_{0}(\mathfrak{m})$, allows us to construct an eigenvariety $\mathscr{E}(\mathfrak{n}, \mathfrak{m})$ for the triple $\left(\mathscr{H}(\mathfrak{m} \mathfrak{n}), \mathscr{Z}(\mathfrak{n}, \mathfrak{m}), \mathscr{W}_{\kappa}\right)$. The Zariski density and accumulation properties for the classical points follow from a special case of the classicality criterion of [Loeffler 2011, Theorem 3.9.6]. To obtain the eigenvariety for $\left(\mathscr{H}(\mathfrak{m n}), \mathscr{Z}(\mathfrak{n}, \mathfrak{m})_{\bar{\rho}}, \mathscr{W}_{\kappa}\right)$ we 
just take the union of connected components in $\mathscr{E}(\mathfrak{n}, \mathfrak{m})$ whose closed points have associated residual Galois representation isomorphic to $\bar{\rho}$.

We then define $\mathscr{E}(\mathfrak{n}, \mathfrak{m})_{\bar{\rho}}^{\mathfrak{q} \text {-sp }}$ to be the Zariski closure in $\mathscr{E}(\mathfrak{n}, \mathfrak{m})_{\bar{\rho}}$ of the subset $Z(\mathfrak{n m})_{\bar{\rho}}^{\mathfrak{q}-\mathrm{sp}} \subset Z(\mathfrak{n m})_{\bar{\rho}}$ corresponding to systems of Hecke eigenvalues in $\mathscr{Z}(\mathfrak{n}, \mathfrak{m})_{\bar{\rho}}^{\mathfrak{q} \text {-sp }}$. Similarly, we define $\mathscr{E}(\mathfrak{n}, \mathfrak{m})_{\bar{\rho}}^{\mathfrak{q}-p s}$ to be the Zariski closure in $\mathscr{E}(\mathfrak{n}, \mathfrak{m})_{\bar{\rho}}$ of the subset $Z(\mathfrak{n m})_{\bar{\rho}}^{\mathfrak{q}-p s}$. We now need to check that $\left.Z(\mathfrak{n m})\right)_{\bar{\rho}}^{\mathfrak{q}-s p}$ and $\left.Z(\mathfrak{n m})\right)_{\bar{\rho}}^{\mathfrak{q}-\mathrm{ps}}$ are accumulation subsets in their Zariski closures, together with the rest of the assertions of the theorem.

We can deduce everything we need by applying the results of [Bellaïche and Chenevier 2009, 7.8] on the family of Weil-Deligne representations carried by an eigenvariety (see also [Paulin 2011]). We proceed as follows: Denote by $X \subset \mathscr{E}(\mathfrak{n}, \mathfrak{m})_{\bar{\rho}}$ the reduced closed subspace given by the union of irreducible components where the monodromy operator in the associated family of Weil-Deligne representations is generically nonzero - we call such a component 'generically special'. More precisely, in the notation of [Bellaïche and Chenevier 2009, 7.8], a generically special component $W$ is one in which for all closed points $x \in W$ we have $N_{s(x)}^{\text {gen }}$ nonzero for $s(x)$ any germ of an irreducible component at $x$ which is contained in $W$. Then we claim that

$$
X \cap Z(\mathfrak{n m})_{\bar{\rho}}=Z(\mathfrak{n m})_{\bar{\rho}}^{\mathfrak{q}-\mathrm{sp}} .
$$

Indeed, if $x \in Z(\mathfrak{n m}))_{\bar{\rho}}^{\mathfrak{q}-\text { sp }}$, then, by Proposition 7.8.19(iii) of [Bellaïche and Chenevier 2009] and local-global compatibility at $\mathfrak{q}$ for the automorphic representation $\pi_{x}$ [Carayol 1986b], every irreducible component passing through $x$ is generically special.

Conversely, if $x \in X \cap Z(\mathfrak{n m})_{\bar{\rho}}$, then the Weil-Deligne representation at $\mathfrak{q}$ associated to $\rho_{x}$ is forced to have the form $(W \oplus W(1), N)$, where $W$ is a onedimensional $\overline{k(x)}$-vector space with an unramified action of the Weil group $W_{\mathfrak{q}}$ and $W(1)$ denotes the twist of $W$ by the $p$-adic cyclotomic character ( $N$ could be zero or nonzero). This means that either $x \in Z(\mathfrak{n m})_{\bar{\rho}}^{\mathfrak{q}-s p}$ or the local factor $\pi_{\mathfrak{q}}$ of the automorphic representation $\pi$ associated to $x$ is one-dimensional. The latter situation cannot occur, since $\pi$ is a cuspidal automorphic representation of $\mathrm{GL}_{2}\left(\mathbb{A}_{F}\right)$ and therefore its local factors are infinite-dimensional (this follows from the existence of a global Whittaker model; for example, see the proof of Theorem 11.1 of [Jacquet and Langlands 1970]).

Now it is easy to deduce the accumulation property for $Z(\mathfrak{n m})_{\bar{\rho}}^{\mathfrak{q}-\text { sp }}$ from the accumulation property for $Z(\mathfrak{n m})_{\bar{\rho}}$.

Similar arguments apply if we take $Y \subset \mathscr{E}(\mathfrak{n}, \mathfrak{m})_{\bar{\rho}}$ to be the reduced closed subspace given by the union of irreducible components where the monodromy operator in the associated family of Weil-Deligne representations is generically zero - we call such components 'generically principal series'. 
Finally we need to construct the map

$$
\mathscr{E}(\mathfrak{n}, \mathfrak{m})_{\bar{\rho}}^{\mathfrak{q}-p s} \rightarrow \mathscr{E}(\mathfrak{n}, \mathfrak{m} / \mathfrak{q})_{\bar{\rho}}
$$

This can be done by giving an alternative construction of $\mathscr{E}(\mathfrak{n}, \mathfrak{m})_{\bar{\rho}}^{\mathfrak{q}-p s}$-indeed, by the uniqueness of abstract eigenvarieties, $\mathscr{E}(\mathfrak{n}, \mathfrak{m})_{\bar{\rho}}^{\mathfrak{q}-p s}$ coincides with the nilreduction of the covering of $\mathscr{E}(\mathfrak{n}, \mathfrak{m} / \mathfrak{q})_{\bar{\rho}}$ given by the roots of $X^{2}-\psi\left(T_{\mathfrak{q}}\right) X+N \mathfrak{q} \psi\left(S_{\mathfrak{q}}\right)(x)$ (this has a natural interpretation as a relative spectrum over $\mathscr{E}(\mathfrak{n}, \mathfrak{m} / \mathfrak{q}) \bar{\rho})$.

3.20. Kisin and Lai's eigenvarieties. Now we let $f$ be a Hecke eigenform as in the statement of Proposition 2.3. For each prime ideal $\mathfrak{p} \mid p$, we denote the two distinct roots of $X^{2}-\theta_{f}\left(T_{\mathfrak{p}}\right) X+(N \mathfrak{p})^{1-w^{-1}} \chi_{f}\left(\varpi_{\mathfrak{p}}\right)$ by $\alpha_{\mathfrak{p}}$ and $\beta_{\mathfrak{p}}$. Then for each subset $S \subset\{\mathfrak{p} \mid p\}$ there is a unique Hecke eigenform

$$
f_{S} \in H^{0}\left(\mathscr{M}^{\mathrm{Iw}}(\mathfrak{n}, \mathfrak{q})_{L}, \underline{\omega}^{\kappa}\right),
$$

whose Hecke eigenvalues away from $p$ are the same as $f$, and for $\mathfrak{p} \mid p$ we have $\theta_{f_{S}}\left(U_{\mathfrak{p}}\right)=\alpha_{\mathfrak{p}}$ if $\mathfrak{p} \in S$ and $\theta_{f_{S}}\left(U_{\mathfrak{p}}\right)=\beta_{\mathfrak{p}}$ if $\mathfrak{p} \notin S$.

We moreover define a point $\nu_{S} \in \widehat{T}\left(\overline{\mathbb{Q}}_{p}\right)$ to be given by the character

$$
\bigotimes_{\mathfrak{p} \mid p} \chi_{\mathfrak{p}, 1} \otimes \chi_{\mathfrak{p}, 2} \prod_{\tau \in \Sigma_{\mathfrak{p}}} \tau^{\left(k_{\tau}-2-w\right) / 2} \otimes \tau^{\left(-w-k_{\tau}+2\right) / 2},
$$

where the $\chi_{\mathfrak{p}, i}$ are characters of $F_{\mathfrak{p}}^{\times} / \mathcal{O}_{\mathfrak{p}}^{\times}$defined by:

- $\chi_{\mathfrak{p}, 1}\left(\varpi_{\mathfrak{p}}\right)=\alpha_{\mathfrak{p}}(N \mathfrak{p})^{-1}$ if $\mathfrak{p} \in T$ and $\chi_{\mathfrak{p}, 1}\left(\varpi_{\mathfrak{p}}\right)=\beta_{\mathfrak{p}}(N \mathfrak{p})^{-1}$ otherwise.

- $\chi_{\mathfrak{p}, 2}\left(\varpi_{\mathfrak{p}}\right)=\beta_{\mathfrak{p}}$ if $\mathfrak{p} \in T$ and $\chi_{\mathfrak{p}, 1}\left(\varpi_{\mathfrak{p}}\right)=\alpha_{\mathfrak{p}}$ otherwise.

Proposition 3.21. For each $S \subset\{\mathfrak{p} \mid p\}$ there is a point $x_{S}$ of $\mathscr{E}(\mathfrak{n}, \mathfrak{q l})_{\bar{\rho}}$ such that

$$
v\left(x_{S}\right)=v_{S},
$$

and the character

$$
\psi_{x_{S}}: \mathscr{H}(\mathfrak{n q} \mathfrak{l}) \rightarrow \overline{\mathbb{Q}}_{p}
$$

induced by $\psi$ is compatible with $\theta_{f_{S}}$.

Proof. For this result, we need to use an alternative construction of the eigenvariety $\mathscr{E}(\mathfrak{n}, \mathfrak{q} \mathfrak{l}) \bar{\rho}$. This is given by the space $\mathscr{C}_{\kappa}(\mathfrak{m})$ of [Kisin and Lai 2005 , Theorem 4.5.4] (with modified level structures). Here the $\mathfrak{m}$ corresponds to our choice of residual Galois representation $\bar{\rho}$. To show that $\mathscr{C}_{\kappa}(\mathfrak{m})$ coincides with $\mathscr{E}(\mathfrak{n}, \mathfrak{q} \mathfrak{l}) \bar{\rho}$, we need to verify that the subset of $\mathscr{C}_{\kappa}(\mathfrak{m})$ corresponding to the 'classical points' $\mathscr{Z}\left(\mathfrak{n}, \mathfrak{q} \mathfrak{l}_{\bar{\rho}}\right.$ is Zariski-dense and accumulation. This follows from a classicality criterion for overconvergent Hilbert modular forms, which has recently been proved in two different ways - by Pilloni and Stroh [2011, Théorème 1.2] and by Tian and Xiao [2013, Proposition 6.3]. Note that Theorem 6.5 of the latter is the statement that the classical points are Zariski-dense in the Kisin-Lai eigenvarieties, but the 
accumulation property also follows immediately from the proof. The existence of the points $x_{S}$ is now immediate from the construction of the Kisin-Lai eigenvariety.

Alternatively, one can avoid the appeal to difficult classicality theorems and instead show that $\mathscr{E}(\mathfrak{n}, \mathfrak{q} \mathfrak{l})_{\bar{\rho}}$ is isomorphic (with its additional structures) to the Zariski closure of the classical points in $\mathscr{C}_{\kappa}(\mathfrak{m})$ by working with the set-up described in Remark 3.16. Applying [Kisin and Lai 2005, Theorem 4.5.6] then concludes the proof.

Since the local factor $\pi_{f, \mathfrak{q}}$ is an unramified twist of Steinberg, one naturally expects that the points $x_{S}$ lie in the eigenvariety $\mathscr{E}(\mathfrak{n}, \mathfrak{q} \mathfrak{l})_{\bar{\rho}}^{\mathfrak{q}-s p}$. Proving that this is the case is a little delicate: the first thing to observe is that since we do not yet know local-global compatibility at $\mathfrak{q}$ for $\pi_{f}$, it is also possible that $x_{S}$ could lie in $\mathscr{E}(\mathfrak{n}, \mathfrak{q} \mathfrak{l})_{\bar{\rho}}^{\mathfrak{q}-p s}$.

One can build $\mathscr{E}\left(\mathfrak{n}, \mathfrak{q} l \overline{\mathfrak{q}}_{\bar{\rho}}^{\mathfrak{q}-\mathrm{sp}}\right.$ using modules given by taking a kernel of trace maps to prime-to-q level from the appropriate Banach modules of overconvergent forms. However, forming this kernel (for modules over the ring of functions on an affinoid open $U$ in weight space) does not necessarily commute with base change from $U$ to a point in weight space (the cokernel of the trace maps may not be flat). So the fact that $\pi_{f, \mathfrak{q}}$ is an unramified twist of Steinberg does not immediate imply that $x_{S}$ lies in $\mathscr{E}(\mathfrak{n}, \mathfrak{q} \mathfrak{l}) \bar{\rho}$-sp. One needs to show that $x_{S}$ lies in a family of forms which are 'q-new'. This problem is solved in [Newton 2014]:

Proposition 3.22. The points $x_{S}$ of Proposition 3.21 all lie in $\mathscr{E}(\mathfrak{n}, \mathfrak{q} l)_{\bar{\rho}}^{\mathfrak{q}-s p}$.

Proof. Take a point $x_{S}$ and suppose that it does not lie in $\mathscr{E}(\mathfrak{n}, \mathfrak{q l})_{\bar{\rho}}^{\mathfrak{q} \text {-sp }}$. Then $x_{S}$ is a point of $\mathscr{E}(\mathfrak{n}, \mathfrak{q} l))_{\bar{\rho}}^{\mathfrak{q}-p s}$, so we may consider the image of $x_{S}$ in $\mathscr{E}(\mathfrak{n}, \mathfrak{l}) \bar{\rho}$. This point satisfies the assumptions of [Newton 2014, Theorem 4.3], but the conclusion of this theorem tells us that $x_{S}$ is indeed in $\mathscr{E}(\mathfrak{n}, \mathfrak{q} \mathfrak{l})_{\bar{\rho}}^{\mathfrak{q}-s p}$.

\section{Completed cohomology and level optimisation}

We give another construction of the eigenvariety $\mathscr{E}(\mathfrak{n}, \mathfrak{m})_{\bar{\rho}}^{\mathfrak{q} \text {-sp }}$, using the completed cohomology of Shimura curves. We will then apply [Newton 2013, Theorem 4.33] to deduce the following result:

Theorem 4.1. Let $S \subset\{\mathfrak{p} \mid p\}$ and let $x_{S}$ be the point of $\mathscr{E}(\mathfrak{n}, \mathfrak{q} \mathfrak{l}) \overline{\bar{q}}$-sp obtained from Propositions 3.21 and 3.22. Then there is a point $y_{S}$ of $\mathscr{E}(\mathfrak{n}, \mathfrak{q}) \bar{q}$-sp such that:

- The Hecke eigenvalues outside $\mathfrak{l}$ coincide with those of $x_{S}$.

- $\psi_{x_{S}}\left(U_{\mathfrak{l}}\right)$ is one of the roots of $X^{2}-\psi_{y_{S}}\left(T_{\mathfrak{l}}\right) X+(N \mathfrak{l})^{1-w_{\iota}} \iota^{-1} \chi_{f}\left(\varpi_{\mathfrak{l}}\right)$.

The proof of this theorem occupies the rest of the section. 
4.2. Completed cohomology of Shimura curves. For this section, we fix a quaternion algebra $B / F$ such that $B$ is nonsplit at precisely one infinite place, denoted $\tau_{1}$, and one finite place, denoted $\mathfrak{q}$ (recall that $[F: \mathbb{Q}]$ is assumed to be even, so such quaternion algebras exist). Denote by $G_{B}$ the reductive algebraic group over $\mathbb{Q}$ arising from the unit group of $B$. Note that $G_{B}$ is an inner form of $\operatorname{Res}_{F / \mathbb{Q}}\left(\mathrm{GL}_{2}\right)$. For $U$ a compact open subgroup of $G_{B}\left(\mathbb{A}_{f}\right)$, we have a complex (disconnected) Shimura curve

$$
M(U)(\mathbb{C})=G_{B}(\mathbb{Q}) \backslash G_{B}\left(\mathbb{A}_{f}\right) \times(\mathbb{C}-\mathbb{R}) / U,
$$

where $G_{B}(\mathbb{Q})$ acts on $\mathbb{C}-\mathbb{R}$ via the $\tau_{1}$ factor of $G_{B}(\mathbb{R})$.

These curves have canonical models over $F$, which we denote by $M(U)$. We follow the conventions of [Carayol 1986a] to define this canonical model.

Definition 4.3. We define $\widetilde{H}^{1}\left(U^{p}, L\right)$ to be

$$
(\underbrace{\lim }_{n} \underset{U_{p}}{\lim _{\text {êt }}} H_{1}^{1}\left(M\left(U_{p} U^{p}\right)_{\bar{F}}, \mathscr{O}_{L} / \mathfrak{m}_{L}^{n}\right)) \otimes_{O_{L}} L
$$

where $U^{p}$ is any compact open subgroup of $G_{B}\left(\mathbb{A}^{\infty, p}\right)$ and $U_{p}$ runs over the compact open subgroups of $G_{B}\left(\mathbb{Q}_{p}\right)$.

The $L$-vector space $\widetilde{H}^{1}\left(U^{p}, L\right)$ is naturally an $L$-Banach space with an admissible continuous action of $G_{B}\left(\mathbb{Q}_{p}\right) \cong \prod_{\mathfrak{p} \mid p} \mathrm{GL}_{2}\left(F_{\mathfrak{p}}\right)$. Moreover, there is a direct summand $\widetilde{H}^{1}\left(U^{p}, L\right)_{\bar{\rho}}$ such that all the systems of Hecke eigenvalues arising from $\widetilde{H}^{1}\left(U^{p}, L\right)_{\bar{\rho}}$ correspond to Galois representations whose residual representations are isomorphic to $\bar{\rho}$.

We now explain how the systems of Hecke eigenvalues parametrised by the set $\mathscr{Z}(\mathfrak{n}, \mathfrak{m})_{\bar{\rho}}^{\mathfrak{q} \text {-sp }}$ can be seen in the space $\widetilde{H}^{1}\left(U^{p}, L\right)_{\bar{\rho}}$.

Suppose we have a $\Sigma$-tuple of integers $k=\left(k_{\tau}\right)_{\tau \in \Sigma}$ with each $k_{\tau} \geq 2$ and an integer $w$ with $k_{\tau}=w \bmod 2$ for all $\tau$.

We denote by $W_{k, w}$ the $L$-representation of $\operatorname{GL}_{2}\left(F_{p}\right)$ defined by

$$
\otimes_{\tau \in \Sigma_{p}}(\tau \circ \mathrm{det})^{\left(w-k_{\tau}+2\right) / 2} \mathrm{Sym}^{k_{\tau}-2} V_{\tau},
$$

where $V_{\tau}$ is the representation of $\mathrm{GL}_{2}\left(F_{p}\right)$ acting via $\tau$ and the standard representation of $\mathrm{GL}_{2}(L)$. These representations then give rise to lisse étale $L$-sheaves $\mathscr{F}_{k, w}$ on the curves $M(U)$ (see, for example, [Newton 2013, §3.2]).

We set $U^{p}$ to be the prime-to- $p$ part of the compact open subgroup of $G_{B}\left(\mathbb{A}^{\infty}\right)$ given by $U_{1}(\mathfrak{n}) \cap U_{0}(\mathfrak{m} / \mathfrak{q})$. Now the Hecke algebra $\mathscr{H}(\mathfrak{m n})$ acts on $\tilde{H}^{1}\left(U^{p}, L\right)$ as follows: For places $v$ prime to $\mathfrak{q}$, we have a standard action by double coset operators associated to our fixed uniformisers $\varpi_{v}$. For the place $\mathfrak{q}$, we choose a uniformiser $\varpi_{D_{\mathfrak{q}}}$ of the order $\widehat{O}_{D_{\mathfrak{q}}}$ whose reduced norm is equal to the fixed 
uniformiser $\varpi_{\mathfrak{q}}$ of $\sigma_{\mathfrak{q}}$, and let $U_{\mathfrak{q}}$ act on $\widetilde{H}^{1}\left(U^{p}, L\right)$ via the action of $\varpi_{D_{\mathfrak{q}}}$. This definition is explained by the following:

Lemma 4.4. Let $\pi_{\mathfrak{q}}=\mathrm{St} \otimes \mu$ be an unramified twist of the Steinberg representation of $\mathrm{GL}_{2}\left(F_{\mathfrak{q}}\right)$. The local Jacquet-Langlands correspondent $\operatorname{JL}\left(\pi_{\mathfrak{q}}\right)$ of $\pi_{\mathfrak{q}}$ is the onedimensional representation of $D_{\mathfrak{q}}^{\times}$given by $\mu \circ \mathrm{Nrd}$, where $\mathrm{Nrd}$ denotes the reduced norm. The $U_{\mathfrak{q}}$-eigenvalue of the space of Iwahori-invariants in $\pi_{\mathfrak{q}}$ is equal to $\mu\left(\varpi_{\mathfrak{q}}\right)$, and is therefore equal to the eigenvalue for the action of $\varpi_{D_{\mathfrak{q}}}$ on $\operatorname{JL}\left(\pi_{\mathfrak{q}}\right)$.

Proof. This follows from the standard computation of the $U_{\mathfrak{q}}$-eigenvalue of the space of Iwahori-invariants in $\pi_{\mathfrak{q}}$.

We have the following proposition, which is proved exactly the same way as [Newton 2013, Theorem 5.2]:

Proposition 4.5. There is a $G_{B}\left(\mathbb{Q}_{p}\right)$-, $\operatorname{Gal}(\bar{F} / F)$ - and Hecke-equivariant isomorphism

$$
\bigoplus_{(k, w)}{\underset{\lim }{U_{p}}}_{H_{\text {ét }}^{1}}\left(M\left(U_{p} U^{p}\right)_{\bar{F}}, \mathscr{F}_{k, w}\right)_{\bar{\rho}} \otimes_{L} W_{k, w}^{\vee} \cong \tilde{H}^{1}\left(U^{p}, L\right)_{\bar{\rho}}^{\mathrm{alg}},
$$

where the right-hand side is the space of locally algebraic vectors (in the sense of [Emerton 2011, 4.2.6]) in the L-Banach space representation $\widetilde{H}^{1}\left(U^{p}, L\right)_{\bar{\rho}}$.

The above proposition allows us to determine the contribution of classical automorphic representations to the Jacquet module (in the sense of [Emerton 2006a]) $J_{B}\left(\tilde{H}^{1}\left(U^{p}, L\right)_{\bar{\rho}}\right)$ of the $\left(\mathbb{Q}_{p^{-}}\right)$locally analytic vectors in $\tilde{H}^{1}\left(U^{p}, L\right)_{\bar{\rho}}$ :

Lemma 4.6. There is a $T$-, $\operatorname{Gal}(\bar{F} / F)$ - and Hecke-equivariant embedding

$$
\bigoplus_{(k, w)}\left(\underset{U_{p}}{\lim _{\text {ét }}} H_{\text {en }}^{1}\left(M\left(U_{p} U^{p}\right)_{\bar{F}}, \mathscr{F}_{k, w}\right)_{\bar{\rho}}\right)_{N} \otimes_{L} \chi_{k, w} \hookrightarrow J_{B}\left(\tilde{H}^{1}\left(U^{p}, L\right)_{\bar{\rho}}\right),
$$

where the subscript $N$ denotes coinvariants (i.e., the classical Jacquet module) and $\chi_{k, w}$ is the character of $T$ given by

$$
\left(\begin{array}{cc}
s_{1} & 0 \\
0 & s_{2}
\end{array}\right) \mapsto \prod_{\tau \in \Sigma_{p}} \tau\left(s_{1}\right)^{\left(k_{\tau}-2-w\right) / 2} \tau\left(s_{2}\right)^{\left(-w-k_{\tau}+2\right) / 2}
$$

Proof. This follows from left-exactness of the Jacquet module functor and [Emerton 2006a, Proposition 4.3.6], since the highest weight space $\left(W_{k, w}^{\vee}\right)^{N}$ has $T$-action given by $\chi_{k, w}$.

The following lemma is a standard result in the smooth representation theory of the groups $\mathrm{GL}_{2}\left(F_{\mathfrak{p}}\right)$ : 
Lemma 4.7. Let $\mu, \mu_{1}, \mu_{2}$ be smooth complex characters of $F_{\mathfrak{p}}{ }^{\times}$.

(1) The Jacquet module $\pi\left(\mu_{1}, \mu_{2}\right)_{N(\mathfrak{p})}$ is isomorphic as a $T_{\mathfrak{p}}$-representation to

$$
\mu_{1}|\cdot|_{\mathfrak{p}}^{1 / 2} \otimes \mu_{2}|\cdot|_{\mathfrak{p}}^{-1 / 2} \oplus \mu_{2}|\cdot|_{\mathfrak{p}}^{1 / 2} \otimes \mu_{1}|\cdot|_{\mathfrak{p}}^{-1 / 2} .
$$

(2) The Jacquet module $(\mathrm{St} \otimes \mu)_{N(\mathfrak{p})}$ is isomorphic as a $T_{\mathfrak{p}}$-representation to

$$
\mu|\cdot|_{\mathfrak{p}} \otimes \mu|\cdot|_{\mathfrak{p}}^{-1} .
$$

Proof. See for example [Goldfeld and Hundley 2011, Theorem 8.12.15].

As a consequence of Lemma 4.7, together with the Jacquet-Langlands correspondence and the contribution of automorphic representations of $G_{B}(\mathbb{A})$ to the cohomology of the curves $M(U)$, we obtain the following proposition:

Lemma 4.8. Suppose $(\psi, v) \in \mathscr{Z}(\mathfrak{n}, \mathfrak{m})_{\bar{\rho}}^{\mathfrak{q}-\mathrm{sp}}$. Then there is a nonzero element

$$
v \in J_{B}\left(\widetilde{H}^{1}\left(U^{p}, L\right)_{\bar{\rho}}\right) \otimes_{L} \overline{\mathbb{Q}}_{p}
$$

on which the Hecke operators away from $p$ act via the character $\psi$ and on which the torus $T$ acts via the character $v$.

The above lemma tells us that the 'classical set' $\mathscr{Z}(\mathfrak{n}, \mathfrak{m})_{\bar{\rho}}^{\mathfrak{q}-s p}$ can be seen in the locally analytic $T$-representations $J_{B}\left(\widetilde{H}^{1}\left(U^{p}, L\right) \bar{\rho}\right)$. We now summarise Emerton's construction of an eigenvariety from this representation, and show that it is an eigenvariety for the triple $\left(\mathscr{H}(\mathfrak{m n}), \mathscr{Z}(\mathfrak{n}, \mathfrak{m})_{\bar{\rho}}^{\mathfrak{q}-\mathrm{sp}}, \mathscr{W}_{\kappa}\right)$.

The $T$-representation $J_{B}\left(\widetilde{H}^{1}\left(U^{p}, L\right) \bar{\rho}\right)$ is naturally dual to a coherent sheaf $\mathscr{M}$ on $\widehat{T}$ (see [Emerton 2006b, Proposition 2.3.2]). Denote by $Y$ the fibre product $\widehat{T} \times \widehat{T}_{0} \mathscr{W}_{\kappa}$, and let $\mathscr{M}_{Y}$ denote the pullback of $\mathscr{M}$ to a coherent sheaf on $Y$.

Taking the relative spectrum of the commutative subalgebra of endomorphisms of this sheaf generated by the Hecke algebra $\mathscr{H}(\mathfrak{m n})$ gives a rigid space with a finite map to $Y$. Passing to the nilreduction gives a reduced rigid space which we denote by $\mathscr{E}_{K}$. By the above lemma, we have a subset $Z \subset \mathscr{E}_{\kappa}$ of classical points corresponding to the elements of $\mathscr{Z}(\mathfrak{n}, \mathfrak{m})_{\bar{\rho}}^{\mathfrak{q}-s p}$.

Lemma 4.9. The space $\mathscr{E}_{K}$, together with the classical subset $Z$, is an eigenvariety for the triple

$$
\left(\mathscr{H}(\mathfrak{m n}), \mathscr{Z}(\mathfrak{n}, \mathfrak{m})_{\bar{\rho}}^{\mathfrak{q}-\mathrm{sp}}, \mathscr{W}_{\kappa}\right)
$$

Proof. The only condition we have to check is that $Z$ is an accumulation and Zariski-dense subset of $\mathscr{E}_{K}$ - everything else follows from the construction of $\mathscr{E}_{K}$. To prove this, we have to interpret $\mathscr{E}_{K}$ as part of (the nilreduction of) an eigenvariety constructed as in [Buzzard 2007], following [Emerton 2006a, Proposition 4.2.36; Newton 2013, Lemma 5.13]. The proof is slightly involved, the main reason being 
that we can show that eigenvarieties constructed with completed cohomology have nice properties only (a priori) after composing the map to weight space $\widehat{T}_{0}$ with a map corresponding to restriction to a finite-index subgroup of $T_{0}$. This comes about because [Emerton 2006a, Proposition 4.2.36] applies to 'cofree' modules over an Iwasawa algebra, not 'coprojective' modules.

Choose $U_{p} \subset G_{B}\left(\mathbb{Q}_{p}\right)$ a sufficiently small compact open subgroup such that $\tilde{H}^{1}\left(U^{p}, L\right)_{\bar{\rho}}$ is a cofree representation of $U_{p} / \overline{F^{\times} \cap U_{p} U^{p}}$, in the sense of [Newton 2013, Definition 5.7]. Such a $U_{p}$ exists by [Newton 2013, Corollary 5.8] — in fact, it suffices to take $U_{p}$ pro- $p$, since our assumptions on the tame level already ensure that $U_{p} U^{p}$ is neat.

Denoting the closed subgroup $\overline{F^{\times} \cap U_{p} U^{p}}$ of $U_{p}$ by $X$, we define a compact commutative $p$-adic analytic group by $S:=T_{0} \cap U_{p} / X$, and denote the rigid space parametrising its continuous characters by $\widehat{S}$. The characters corresponding to points of $\mathscr{W}_{\kappa}$ are trivial on the units of $F$ with norm 1 , so (possibly shrinking $U_{p}$ if $p=2$ ) these characters are trivial on $X$. Therefore restriction to $T_{0} \cap U_{p}$ gives a map $\mathscr{W}_{\kappa} \rightarrow \widehat{S}$. In fact, the definition of $\mathscr{W}_{\kappa}$ implies that this map is an isomorphism onto its image, which we denote by $\mathscr{W}_{S}$.

We denote by $\widetilde{\mathscr{W}}_{\kappa}$ the preimage of $\mathscr{W}_{S}$ in $\mathscr{W}$. The space $\widetilde{\mathscr{W}}_{\kappa}$ is a finite disjoint union of open discs, whose components are indexed by characters of the finite group $T_{0} / T_{0} \cap U_{p}$. We denote by $\widetilde{Y}$ the fibre product $\widehat{T} \times \widehat{T}_{0} \widetilde{\mathscr{W}}_{\kappa}$, and let $\mathscr{M}_{\tilde{Y}}$ denote the pullback of $\mathscr{M}$ to a coherent sheaf on $\tilde{Y}$. Mimicking the construction of $\mathscr{E}_{K}$, we obtain a rigid space $\widetilde{\mathscr{E}}_{K}$ with a finite map to $\tilde{Y}$, such that $\mathscr{E}_{K}$ is the open and closed subspace of $\widetilde{\mathscr{E}}_{\kappa}$ lying over $\mathscr{W}_{\kappa}$.

Consider an increasing sequence $X_{n}=\operatorname{Sp}\left(A_{n}\right)$ of admissible affinoid opens covering $\widehat{S}$, and write $M$ for the space of global sections of $\mathscr{M}$ and $M_{n}$ for the base change $M \hat{\otimes}_{\mathscr{C} \text { an }(\widehat{S}, L)} A_{n}$. It follows from [Emerton 2006a, Proposition 4.2.36] that $M_{n}$ is the finite-slope part of an orthonormalisable Banach $A_{n}$-module with the action of a compact operator (coming from the action of an element $z \in T$ ). We may choose the $X_{n}$ such that their inverse images in $\widetilde{\mathscr{W}}_{\kappa}$ are admissible affinoid opens $\tilde{Y}_{n}=\operatorname{Sp}\left(B_{n}\right)$ (e.g., closed discs). It follows from [Buzzard 2007, Corollary 2.10] that the modules $M \hat{\otimes}_{\mathscr{C} \text { an }(\widehat{S}, L)} B_{n}$ are likewise finite-slope parts of orthonormalisable Banach $B_{n}$-modules with the action of a compact operator.

It now follows, as in [Chenevier 2004, Corollaire 6.4.4], that the image of each irreducible component of $\widetilde{\mathscr{E}}_{K}$ in $\mathscr{W}_{S}$ is the image of a Fredholm hypersurface, and is therefore Zariski-open in $\mathscr{W}_{S}$. For each irreducible component of $\widetilde{\mathscr{E}}_{K}$, the map to $\mathscr{W}_{S}$ factors through one of the connected components of $\widetilde{\mathscr{W}}_{\kappa}$, so each irreducible component of $\widetilde{\mathscr{E}}_{K}$ has Zariski-open image in this connected component. In particular, each irreducible component of $\mathscr{E}_{K}$ has Zariski-open image in $\mathscr{W}_{K}$.

The classicality criterion of [Emerton 2006a, Theorem 4.4.5] now shows that $Z$ is Zariski-dense and accumulation in $\mathscr{E}_{K}$. 
Now that we know that $\mathscr{E}(\mathfrak{n}, \mathfrak{m}) \bar{\rho}_{\bar{\rho}}^{\mathfrak{q} \text {-sp }}$ and $\mathscr{E}_{\mathcal{K}}$ are eigenvarieties for the same triple, we have the following:

Corollary 4.10. The closed points $x \in \mathscr{E}(\mathfrak{n}, \mathfrak{m})_{\bar{\rho}}^{\mathfrak{q} \text {-sp }}$ with $v(x)=\lambda \in \widehat{T}\left(\overline{\mathbb{Q}}_{p}\right)$ correspond bijectively with systems of Hecke eigenvalues appearing in the (finitedimensional) $\overline{\mathbb{Q}}_{p}$-vector space

$$
J_{B}\left(\tilde{H}^{1}\left(U^{p}, L\right)_{\bar{\rho}}\right) \otimes_{L} \overline{\mathbb{Q}}_{p}[\lambda],
$$

defined to be the subspace where $T$ acts via the character $\lambda$.

Proof. This follows from the construction of $\mathscr{E}_{K}$ and Lemma 4.9.

Now Theorem 4.1 follows from combining Corollary 4.10 and [Newton 2013, Theorem 4.33].

\section{Proof of Theorem 1.4}

We can also construct $\mathscr{E}(\mathfrak{n}, \mathfrak{q})_{\bar{\rho}}$ using the overconvergent Hilbert modular forms of [Kisin and Lai 2005]. Therefore we conclude from Theorem 4.1 that there are overconvergent Hilbert modular eigenforms $g_{S} \in M_{\mathcal{K}}^{\dagger}(\mathfrak{n}, \mathfrak{q})$ whose systems of Hecke eigenvalues correspond to those of the points $y_{S}$.

Applying [Kassaei 2013, Theorem 7.10], we glue the $g_{S}$ into a classical Hilbert modular eigenform

$$
g \in H^{0}\left(\mathscr{M}(\mathfrak{n}, \mathfrak{q})_{L}, \underline{\omega}^{\kappa}\right)
$$

as in the statement of Proposition 2.3 (we are using more general tame levels than Kassaei, but this presents no problem). This completes the proof of Proposition 2.3, and hence of Theorem 1.4.

Remark 5.1. Note that the situation in the above proof is that we have an overconvergent form $g_{S}$ with level prime to $\mathfrak{l}$ such that one of its $\mathfrak{l}$-stabilisations is equal to the classical form $f_{S}$. One might guess that $g_{S}$ can be obtained from $f_{S}$ by applying a trace map, in which case it is immediate that $g_{S}$ is classical. However, in the case we are considering all the trace maps vanish on $f_{S}$.

A simple example illustrating that some argument is required here is given by the classical weight-two Eisenstein series of level $\Gamma_{0}(l)$ (note that our level-lowering result [Newton 2013, Theorem 4.33] does not apply to this form). It is in the kernel of the trace maps, but it is also the $l$-stabilisation of a (nonoverconvergent) $p$-adic modular form.

\section{Acknowledgements}

During the period in which this research was conducted, the author was supported by Trinity College, Cambridge and an EPSRC postdoctoral fellowship. The author would also like to thank the anonymous referees for their helpful comments. 


\section{References}

[Ash and Stevens 2008] A. Ash and G. Stevens, " $p$-adic deformations of arithmetic cohomology", preprint, 2008, http://www2.bc.edu/ ashav/Papers/Ash-Stevens-Oct-07-DRAFT-copy.pdf.

[Bellaïche and Chenevier 2009] J. Bellaïche and G. Chenevier, Families of Galois representations and Selmer groups, Astérisque 324, Société Mathématique de France, Paris, 2009. MR 2011m:11105 Zbl 1192.11035

[Blasius and Rogawski 1993] D. Blasius and J. D. Rogawski, "Motives for Hilbert modular forms", Invent. Math. 114:1 (1993), 55-87. MR 94i:11033 Zbl 0829.11028

[Buzzard 2004] K. Buzzard, "On p-adic families of automorphic forms", pp. 23-44 in Modular curves and abelian varieties, edited by J. Cremona et al., Progr. Math. 224, Birkhäuser, Basel, 2004. MR 2005g:11069 Zbl 1166.11322

[Buzzard 2007] K. Buzzard, "Eigenvarieties", pp. 59-120 in L-functions and Galois representations, edited by D. Burns et al., London Math. Soc. Lecture Note Ser. 320, Cambridge University Press, 2007. MR 2010g:11076 Zbl 1230.11054

[Carayol 1986a] H. Carayol, "Sur la mauvaise réduction des courbes de Shimura", Compositio Math. 59:2 (1986), 151-230. MR 88a:11058 Zbl 0607.14021

[Carayol 1986b] H. Carayol, "Sur les représentations $l$-adiques associées aux formes modulaires de Hilbert”, Ann. Sci. École Norm. Sup. (4) 19:3 (1986), 409-468. MR 89c:11083 Zbl 0616.10025

[Chenevier 2004] G. Chenevier, "Familles $p$-adiques de formes automorphes pour GLn", J. Reine Angew. Math. 570 (2004), 143-217. MR 2006b:11046 Zbl 1093.11036

[Chenevier 2009] G. Chenevier, "Une application des variétés de Hecke des groups unitaires", preprint, 2009, http://gaetan.chenevier.perso.math.cnrs.fr/articles/famgal.pdf.

[Conrad 1999] B. Conrad, "Irreducible components of rigid spaces", Ann. Inst. Fourier (Grenoble) 49:2 (1999), 473-541. MR 2001c:14045 Zbl 0928.32011

[Emerton 2006a] M. Emerton, "Jacquet modules of locally analytic representations of $p$-adic reductive groups, I: Construction and first properties”, Ann. Sci. École Norm. Sup. (4) 39:5 (2006), 775-839. MR 2008c:22013 Zbl 1117.22008

[Emerton 2006b] M. Emerton, "On the interpolation of systems of eigenvalues attached to automorphic Hecke eigenforms”, Invent. Math. 164:1 (2006), 1-84. MR 2007k:22018 Zbl 1090.22008

[Emerton 2011] M. Emerton, "Locally analytic vectors in representations of locally $p$-adic analytic groups", preprint, 2011, http://www.math.uchicago.edu/ emerton/pdffiles/analytic.pdf. To appear in Memoirs of the AMS.

[Goldfeld and Hundley 2011] D. Goldfeld and J. Hundley, Automorphic representations and Lfunctions for the general linear group, I, Cambridge Studies in Advanced Mathematics 129, Cambridge University Press, 2011. MR 2012i:11054 Zbl 1247.11070

[Jacquet and Langlands 1970] H. Jacquet and R. P. Langlands, Automorphic forms on GL(2), Lecture Notes in Mathematics 114, Springer, Berlin-New York, 1970. MR 53 \#5481 Zbl 0236.12010

[Jarvis 1997] F. Jarvis, "On Galois representations associated to Hilbert modular forms", J. Reine Angew. Math. 491 (1997), 199-216. MR 98h:11067 Zbl 0914.11025

[Jorza 2010] A. Jorza, Crystalline representations for GL(2) over quadratic imaginary fields, Ph.D. thesis, Princeton University, 2010, http://search.proquest.com/docview/522118786. MR 2753218

[Kassaei 2013] P. L. Kassaei, "Modularity lifting in parallel weight one”, J. Amer. Math. Soc. 26:1 (2013), 199-225. MR 2983010 Zbl 1296.11052 
[Kassaei et al. 2014] P. L. Kassaei, S. Sasaki, and Y. Tian, "Modularity lifting results in parallel weight one and applications to the Artin conjecture: the tamely ramified case", Forum Math. Sigma 2 (2014), 58 (electronic). MR 3294624 Zbl 1296.11053

[Kisin and Lai 2005] M. Kisin and K. F. Lai, "Overconvergent Hilbert modular forms", Amer. J. Math. 127:4 (2005), 735-783. MR 2006d:11045 Zbl 1129.11020

[Loeffler 2011] D. Loeffler, "Overconvergent algebraic automorphic forms", Proc. Lond. Math. Soc. (3) 102:2 (2011), 193-228. MR 2012f:11098 Zbl 1232.11056

[Luu 2015] M. Luu, Deformation theory and local-global compatibility of Langlands correspondences, Memoirs of the AMS 1123, Amer. Math. Soc., Providence, RI, online publication February 2015.

[Moy and Specter 2015] R. Moy and J. Specter, "There exist non-CM Hilbert modular forms of partial weight 1", preprint, 2015. arXiv 1407.3872

[Newton 2013] J. Newton, "Completed cohomology of Shimura curves and a $p$-adic JacquetLanglands correspondence", Math. Ann. 355:2 (2013), 729-763. MR 3010145 Zbl 1304.22025

[Newton 2014] J. Newton, "Level raising for $p$-adic Hilbert modular forms", preprint, 2014. arXiv 1409.6533

[Paulin 2011] A. G. M. Paulin, "Local to global compatibility on the eigencurve", Proc. Lond. Math. Soc. (3) 103:3 (2011), 405-440. MR 2012i:11047 Zbl 1290.11080

[Pilloni and Stroh 2011] V. Pilloni and B. Stroh, "Surconvergence et classicité: le cas Hilbert", preprint, 2011, http://perso.ens-lyon.fr/vincent.pilloni/surconv_hilbert.pdf.

[Pilloni and Stroh 2013] V. Pilloni and B. Stroh, "Surconvergence, ramification et modularité", preprint, 2013, http://perso.ens-lyon.fr/vincent.pilloni/Artinfinal.pdf.

[Ribet 1977] K. A. Ribet, "Galois representations attached to eigenforms with Nebentypus", pp. 17-51 in Modular functions of one variable, V (Univ. Bonn, 1976), edited by J.-P. Serre and D. Zagier, Lecture Notes in Mathematics 601, Springer, Berlin, 1977. MR 56 \#11907 Zbl 0363.10015

[Rogawski and Tunnell 1983] J. D. Rogawski and J. B. Tunnell, "On Artin $L$-functions associated to Hilbert modular forms of weight one", Invent. Math. 74:1 (1983), 1-42. MR 85i:11044 Zbl 0523.12009

[Sasaki 2014] S. Sasaki, "Integral models of Hilbert modular varieties in the ramified case, deformations of modular Galois representations, and weight one forms", preprint, 2014, http:// www.cantabgold.net/users/s.sasaki.03/hmv1-3-14.pdf.

[Taylor 1989] R. Taylor, "On Galois representations associated to Hilbert modular forms", Invent. Math. 98:2 (1989), 265-280. MR 90m:11176 Zbl 0705.11031

[Taylor 1995] R. Taylor, "On Galois representations associated to Hilbert modular forms, II", pp. 185-191 in Elliptic curves, modular forms \& Fermat's last theorem (Hong Kong, 1993), edited by J. Coates, Ser. Number Theory I, Int. Press, Cambridge, MA, 1995. MR 96j:11073 Zbl 0836.11017

[Tian and Xiao 2013] Y. Tian and L. Xiao, " $p$-adic cohomology and classicality of overconvergent Hilbert modular forms”, preprint, 2013. arXiv 1308.0779

Communicated by Brian Conrad

Received 2014-09-24 Revised 2015-02-06 Accepted 2015-03-27

j.newton@imperial.ac.uk Department of Mathematics, Imperial College London, South Kensington Campus, London SW7 2AZ, United Kingdom 


\section{Algebra \& Number Theory}

msp.org/ant

\section{EDITORS}

MANAGING EDITOR

Bjorn Poonen

Massachusetts Institute of Technology

Cambridge, USA

\author{
EDITORIAL BOARD CHAIR \\ David Eisenbud \\ University of California \\ Berkeley, USA
}

\section{BOARD OF EDITORS}

Georgia Benkart

Dave Benson

Richard E. Borcherds

John H. Coates

J-L. Colliot-Thélène

Brian D. Conrad

Hélène Esnault

Hubert Flenner

Edward Frenkel

Andrew Granville

Joseph Gubeladze

Roger Heath-Brown

Craig Huneke

Kiran S. Kedlaya

János Kollár

Yuri Manin

Barry Mazur

Philippe Michel
University of Wisconsin, Madison, USA

University of Aberdeen, Scotland

University of California, Berkeley, USA

University of Cambridge, UK

CNRS, Université Paris-Sud, France

Stanford University, USA

Freie Universität Berlin, Germany

Ruhr-Universität, Germany

University of California, Berkeley, USA

Université de Montréal, Canada

San Francisco State University, USA

Oxford University, UK

University of Virginia, USA

Univ. of California, San Diego, USA

Princeton University, USA

Northwestern University, USA

Harvard University, USA

École Polytechnique Fédérale de Lausanne
Susan Montgomery

Shigefumi Mori

Raman Parimala

Jonathan Pila

Anand Pillay

Victor Reiner

Peter Sarnak

Joseph H. Silverman

Michael Singer

Vasudevan Srinivas

J. Toby Stafford

Ravi Vakil

Michel van den Bergh

Marie-France Vignéras

Kei-Ichi Watanabe

Efim Zelmanov

Shou-Wu Zhang
University of Southern California, USA

RIMS, Kyoto University, Japan

Emory University, USA

University of Oxford, UK

University of Notre Dame, USA

University of Minnesota, USA

Princeton University, USA

Brown University, USA

North Carolina State University, USA

Tata Inst. of Fund. Research, India

University of Michigan, USA

Stanford University, USA

Hasselt University, Belgium

Université Paris VII, France

Nihon University, Japan

University of California, San Diego, USA

Princeton University, USA

\section{PRODUCTION}

production@msp.org

Silvio Levy, Scientific Editor

See inside back cover or msp.org/ant for submission instructions.

The subscription price for 2015 is US $\$ 255 /$ year for the electronic version, and $\$ 440 /$ year ( $+\$ 55$, if shipping outside the US) for print and electronic. Subscriptions, requests for back issues and changes of subscribers address should be sent to MSP.

Algebra \& Number Theory (ISSN 1944-7833 electronic, 1937-0652 printed) at Mathematical Sciences Publishers, 798 Evans Hall \#3840, c/o University of California, Berkeley, CA 94720-3840 is published continuously online. Periodical rate postage paid at Berkeley, CA 94704, and additional mailing offices.

ANT peer review and production are managed by EditFLOW ${ }^{\circledR}$ from MSP.

\section{PUBLISHED BY}

- mathematical sciences publishers

nonprofit scientific publishing

http://msp.org/

(C) 2015 Mathematical Sciences Publishers 


\section{Algebra \& Number Theory}

Volume $9 \quad$ No. $4 \quad 2015$

Motivic Donaldson-Thomas invariants of small crepant resolutions

ANDREW MORRISON and KENTARO NAGAO

Étale homotopy equivalence of rational points on algebraic varieties

AMBRUS PÁL

Fermat's last theorem over some small real quadratic fields

NUNO FREITAS and SAMIR SIKSEK

Bounded negativity of self-intersection numbers of Shimura curves in Shimura surfaces MARTIN MÖLLER and DOMINGo TOLEDO

Singularities of locally acyclic cluster algebras

Angélica Benito, Greg Muller, Jenna Rajchgot and Karen E. Smith

On an analytic version of Lazard's isomorphism

GEORG TAMME

Towards local-global compatibility for Hilbert modular forms of low weight JAMES NEWTON

Horrocks correspondence on arithmetically Cohen-Macaulay varieties

981

Francesco Malaspina and A. Prabhakar RaO

The Elliott-Halberstam conjecture implies the Vinogradov least quadratic nonresidue conjecture

TERENCE TAO 